\title{
How to choose a delegation for a peace conference?
}

Citation for published version (APA):

Can, B., Csóka, P., \& Ergin, E. (2017). How to choose a delegation for a peace conference? Maastricht University, Graduate School of Business and Economics. GSBE Research Memoranda No. 008 https://doi.org/10.26481/umagsb.2017008

Document status and date:

Published: 06/04/2017

DOI:

10.26481/umagsb.2017008

Document Version:

Publisher's PDF, also known as Version of record

\section{Please check the document version of this publication:}

- A submitted manuscript is the version of the article upon submission and before peer-review. There can be important differences between the submitted version and the official published version of record.

People interested in the research are advised to contact the author for the final version of the publication, or visit the DOI to the publisher's website.

- The final author version and the galley proof are versions of the publication after peer review.

- The final published version features the final layout of the paper including the volume, issue and page numbers.

Link to publication

\footnotetext{
General rights rights.

- You may freely distribute the URL identifying the publication in the public portal. please follow below link for the End User Agreement:

www.umlib.nl/taverne-license

Take down policy

If you believe that this document breaches copyright please contact us at:

repository@maastrichtuniversity.nl

providing details and we will investigate your claim.
}

Copyright and moral rights for the publications made accessible in the public portal are retained by the authors and/or other copyright owners and it is a condition of accessing publications that users recognise and abide by the legal requirements associated with these

- Users may download and print one copy of any publication from the public portal for the purpose of private study or research.

- You may not further distribute the material or use it for any profit-making activity or commercial gain

If the publication is distributed under the terms of Article $25 \mathrm{fa}$ of the Dutch Copyright Act, indicated by the "Taverne" license above, 


\section{Maastricht University}

Burak Can, Péter Csóka, Emre Ergin

How to choose a delegation for a peace conference?

$\mathrm{RM} / 17 / 008$

\section{GSBE}

Maastricht University School of Business and Economics

Graduate School of Business and Economics

P.O Box 616

NL- 6200 MD Maastricht

The Netherlands 


\title{
How to choose a delegation for a peace conference?
}

\author{
Burak Can* Péter Csóka ${ }^{\dagger} \quad$ Emre Ergin ${ }^{\ddagger}$
}

April 4, 2017

\begin{abstract}
This paper analyzes how to choose a delegation, a committee to represent a society such as in a peace conference. We propose normative conditions and seek optimal, consistent, neutral and non-manipulable ways to choose a delegation. We show that a novel class of threshold rules are characterized by these criteria. The rules impose that a delegation is chosen when its combined support in the society first reaches a particular percentage of the public opinion - depending on the size of the delegation. Conversely, minority opinions that are not reflected in the delegation should always be below a threshold, which follows a geometric series.

Keywords: Aggregation Rules, Committee Selection, Conflict Management, Kemeny Distance, Strategy-proofness.

JEL Classification: C70, D71
\end{abstract}

\footnotetext{
*Maastricht University, Department of Economics. E-mail: b.can@maastrichtuniversity.nl. This work is partly financed by the Netherlands Organisation for Scientific Research (NWO) under the grant with project nr. 451-13017 (VENI, 2014). The support of the NWO, therefore, is gratefully acknowledged.

†Corvinus University of Budapest, Corvinus Business School, Department of Finance and "Momentum" Game Theory Research Group, Centre for Economic and Regional Studies, Hungarian Academy of Sciences. E-mail: peter.csoka@uni-corvinus.hu. Péter Csóka was supported by the ÚNKP-16-4-IV New National Excellence Program of the Ministry of Human Capacities.

${ }^{\ddagger}$ Maastricht University, Department of Economics. E-mail: e.ergin@maastrichtuniversity.nl.
} 


\section{Introduction}

In many situations, individuals participate in collective decision making via a committee of representatives or delegates, i.e., a multi-layered aggregation of individual opinions. Consider, for instance, voting for candidates in elections to represent your opinion in representative democracies. Those candidates, in turn, make decisions with your opinion in mind. Correspondingly, peace conferences and negotiations over conflict zones require delegates to be sent by different interest groups or ethnicities involved in a civil war. The choice of which interest group, ethnicity, etc. to invite to the conference, however, may not be very straightforward. Especially not in extremely heterogenous, polarized and/or divided societies.

With a peace conference scenario in mind, consider a society and issues it is facing. Thinking of individual opinions (priority orderings) over these issues, we want to find out what limited set of opinions we could choose to represent this society in a reasonable, fair way. Eventually, we might bring together some people representing those opinions on behalf of the possibly very diverse interest groups and hope to achieve consensus in the society. We are not interested per se in how much relative importance an opinion has, but only in whether an opinion is relevant enough ${ }^{1}$ to be invited to the table. The mechanism through which this table is formed should preferably respect some minimal normative requirements. For instance, it should respect unanimous agreements in the society and be consistent in choosing delegations for when similar societies are merged. We expect it to be neutral in the way it treats the opinions, and also expect it to be non-manipulable. We propose all these norms as criteria to choose who should get a seat at the table and then show that there is a unique class of rules which satisfy all these criteria, hence a characterization result. These novel rules are not trivial, and relatively simple to comprehend, making them practically usable to set the table for peace negotiations.

Our framework assumes individuals to have preferences/rankings over some available issues, also known as a preference profile of a society. We formalize the delegation rules as systems that assign preferences ${ }^{2}$ to each given preference profile. We then propose conditions on how to choose delegates for this society. We first require that if all individuals in a society agree on how to rank one issue over another, the delegation should respect that. This is also known as Pareto optimality. Second, we impose that when two distinct societies represented by identical delegations merge, the merged society should also be represented by the same delegation (Young, 1974, 1975; Smith, 1973), an idea known as Consistency ${ }^{3}$. Our third condition, Ballot neutrality

\footnotetext{
${ }^{1}$ Of course in principle, plurality requires that every opinion should be included in the peace talks. This is perhaps preferable when there is a limited number of available opinions. However, in cases where the number of ethnicities/interest groups are numerous, it becomes implausible to invite all as delegates to the table. For more on inclusivity of peace negotiations see Dudouet and Lundström (2016); Planta et al. (2015); Papagianni (2014) and Lanz (2011).

${ }^{2}$ Since the delegation does not necessarily comprise of a single ranking, a delegation rule herein corresponds to a social welfare correspondence instead of a social welfare function. However, we employ the term delegation as it entails a particular interpretation.

${ }^{3}$ Consistency additionally implies that the delegation choice is anonymous, a condition which requires that the names of the individuals do not matter.
} 
reflects an idea of fairness, and requires that only the ballots of individual opinions should matter in the delegation choice ${ }^{4}$. Finally, we require that no individual can manipulate the choice of delegates to their advantage. This condition is called Strategy-proofness. The first two, Pareto optimality and consistency, are very standard conditions in the literature. In what follows we explain further ballot neutrality and strategy-proofness.

Ballot neutrality, imposes neutrality towards "equivalent ballots". Consider two societies of equal size, say 6 individuals, facing three issues, hence six possible preferences. Now represent the opinions in both societies by the number of followers each preference has, e.g., $(\mathbf{3}, 2,1,0,0,0)$ and $(0,1,2, \mathbf{3}, 0,0)$. As it happens, the two societies have "equivalent" ballots, i.e., the support distribution is merely a shuffling of the number of followers. In this case, we require the delegation choice in each society to correspond to the support for the delegates. For instance, if the first preference in the former ballot with a support of 3 , is chosen as a delegate in the former society then the fourth preferences in the latter ballot should also be chosen in the second society. Ballot neutrality is also a variable alternative axiom which necessitates that increasing the number of available issues, and preferences, does not influence the outcome so long as the distributions of supported opinions are equivalent ${ }^{5}$. We explain this further in detail in the coming section and provide an example in the appendix.

Strategy-proofness requires that the rule is not manipulable by individuals (or coalitions). Therefore a rule being strategy-proof naturally induces honest reporting of individual opinions. Consider a society and a delegation representing it. Suppose an individual misreports his opinion and this alters the delegation such that at least one new delegate is strictly closer $^{6}$ to his opinion than any other delegate in the original delegation. This situation is considered as a successful manipulation. We require that the delegation choice should not be prone to any such manipulation. If a rule is not manipulable by any individual then we call this condition as individual strategy-proofness, whereas if no coalition of individuals can achieve such manipulation, we call this as coalitional strategy-proofness. The latter is a stronger requirement than the former. ${ }^{7}$

We find that there exists a non-dictatorial, non-trivial and in fact, simple class of rules which is characterized by these conditions, and which we call as threshold rules. All these rules share

\footnotetext{
${ }^{4}$ Ballot neutrality additionally implies that the delegation choice is neutral, a condition which requires that the names of the issues do not matter.

${ }^{5}$ For instance, increasing the number of issues to 4 , and hence the number of possible preferences to 24 , would still yield $R_{1}$ as a delegate if the ballot stayed as $(\mathbf{3}, 2,1,0,0, \ldots, 0)$.

${ }^{6}$ We use the most typical measure of closeness for rankings, i.e., the Kemeny distance (Kemeny, 1959).

${ }^{7}$ When preferences are aggregated into a single alternative, i.e., social choice functions, Gibbard (1973) and Satterthwaite (1975), shows the impossibility of finding such proper non-dictatorial and strategy-proof rules. See Barberà (2011) for more on strategy-proof social choice functions. For strategy proofness of multi-valued social choice rules, see Barberà et al. (2001). When preferences are aggregated into a single ranking, i.e., social welfare functions, the results are mixed since the definition of strategy-proofness can be quite numerous. Bossert and Storcken (1992) proves an impossibility result, Sato (2013) offers more positive news, and finally Bossert and Sprumont (2014) uses a weaker version of strategy-proofness than in Bossert and Storcken (1992) and provides some examples of non-manipulable rules. Our strategy-proofness concept is essentially different than that of Bossert and Storcken (1992) and Athanasoglou (2016) since we allow multiple preferences in the outcome.
} 
a common lower bound in terms of how much representative power they require for all possible sizes of delegations. For instance, if a delegation is composed of $t$ delegates, then the ratio of the individuals supporting those delegates to the whole society must be strictly higher than $1-0.5^{t}$.

The delegation rules we characterize only differ in "how high" the thresholds are set above the common lower bound. For each threshold rule, there exists a threshold function $f$ which imposes how much minimal support a delegation of size $t$ has to have to be an appropriate representation for a society. The rule orders each possible ranking/delegate according to their support in the society and chooses the lowest number of delegates $t^{*}$ with a total support reaching the respective threshold, i.e., $f\left(t^{*}\right)$. For example, a threshold rule might require $60 \%$ of the society's support for singleton delegations, i.e., $f(1)$. If this support is not found, then it might look for $85 \%$ of the society's support for a delegation of size $2, f(2)$. If this support is not found, then the process continues, with monotonically increasing thresholds for each $t$. We show that all threshold rules satisfy two conditions: i) $f(1)>0.5$ and ii) $1 \geq f(t) \geq(f(t-1)+1) / 2$ for $t>2$, i.e.,minimal threshold for a singleton delegation is above $50 \%$, and the minimal threshold for delegations of size $t$ is at least the average of the previous level, $f(t-1)$ and $100 \%$, but at most $100 \%$. Of course, $f(1)$ can also start from $100 \%$ (and hence continue at that level), requiring $100 \%$ support for each possible sizes of delegations, which can only be reached by including all the reported preferences. Threshold rules are well-defined, as the threshold for $t$ sooner or later reaches $100 \%$.

As Lanz (2011) argues, "Only stakeholders who add value to the process and augment the chances of reaching a sustainable settlement should be given seats at the table, [...]". The challenge, therefore, is to make the invitations to the table from a normative perspective while maintaining inclusivity and feasibility. This paper proposes a quantitative measure on how to set the table for invitations, the number of seats at the table, and finally how representative in total, the invitees must minimally be.

The paper proceeds as follows. Section 2 presents the notation and conditions. In Section 3, we define threshold rules and provide some examples. In Section 4, we provide our characterization. Section 5 concludes with some policy implication.

\section{Basic Notation}

\section{1 $\quad$ Model}

Let $\mathcal{A}$ be a countably infinite set of alternatives, interpreted as potential issues. Given a finite nonempty subset $A \subsetneq \mathcal{A}$, preferences are taken to be strict priority rankings of these issues, formalized as complete, antisymmetric and transitive binary relations over the set of alternatives $A$. We denote the set of all preferences over $A$ by $\mathcal{L}(A)$. Given a preference $R \in \mathcal{L}(A)$, and two disjoint alternatives $a$ and $b$, the case where $a$ is preferred to $b$ can be denoted by $R=. a . b$. or $(a, b) \in R$. To measure closeness we use the well-known Kemeny distance. ${ }^{8}$ Formally, for two preferences $R_{1}$ and $R_{2}$, the Kemeny distance is $\delta\left(R_{1}, R_{2}\right)=\left(\left|R_{2} \backslash R_{1}\right|+\left|R_{1} \backslash R_{2}\right|\right) / 2$, half of the

\footnotetext{
${ }^{8}$ Kemeny (1959) introduced this distance. For a recent, improved characterization, see Can and Storcken (2013).
} 
symmetric set difference.

Let $\mathcal{N}$ be a countably infinite set of agents, interpreted as potential individuals. Given a finite nonempty subset $N \subsetneq \mathcal{N}$ with cardinality $n, \mathcal{L}(A)^{n}$ denotes the set of all preference profiles $P$, i.e., preferences of $n$ agents where $P(i)$ refers to the preference of agent $i \in N$ and $P(S)$ refers to the preference profile, say a subprofile, of a subset of agents $S \subseteq N$. Given a profile $P \in \mathcal{L}(A)^{n}$, and $R \in \mathcal{L}(A)$, we denote the number of agents who reported $R$ in this profile as $p(R)=|\{i \in N \mid P(i)=R\}|$.

Given any finite $A \subsetneq \mathcal{A}$, let $R_{1}, R_{2}, \ldots, R_{|A|}$ ! be an enumeration of preferences in $\mathcal{L}(A)$, e.g., the lexicographic enumeration for $A=\{a, b, c\}$ is " $R_{1}=a b c, R_{2}=a c b, R_{3}=b a c, R_{4}=b c a, R_{5}=$ $c a b, R_{6}=c b a$ ". Given any such enumeration, a profile $P \in \mathcal{L}(A)^{n}$ can also be interpreted as a vector composed of the number of followers each preference has, e.g., $p=\left(p_{1}, p_{2},, p_{3}, \ldots, p_{|A| !}\right)$ on $\mathbb{N}^{|A| !}$ with the interpretation that $p_{t}=\left|\left\{i \in N \mid P(i)=R_{t}\right\}\right|$ is the support for preference $R_{t} \in \mathcal{L}(A)$ and $p$ is the support for the preference profile $P$. As an example, for 3 alternatives and the enumeration given above, the support for the following preference profile,

$$
P \in \mathcal{L}(A)^{6}=\{\underbrace{a b c, a b c, a b c}_{R_{1}}, \underbrace{b a c, b a c}_{R_{3}}, \underbrace{c a b}_{R_{5}}\}
$$

can be denoted by $p=(3,0,2,0,1,0)$. For simplicity, we also denote the "normalized support" for the same profile similarly, e.g., $p=(0.5,0,0 . \overline{3}, 0,0.1 \overline{6}, 0)$.

Consider two disjoint finite sets of agents $N, N^{\prime}$, and preference profiles $P \in \mathcal{L}(A)^{n}$, and $P^{\prime} \in \mathcal{L}(A)^{n^{\prime}}$. Then, $\bar{P}=\left(P, P^{\prime}\right) \in \mathcal{L}(A)^{n+n^{\prime}}$ denotes the merging of two profiles, i.e, $\bar{P}(i)=P(i)$ if $i \in N$ and $\bar{P}(i)=P^{\prime}(i)$ if $i \in N^{\prime}$. If $P$ and $P^{\prime}$ are such that there exists a bijection $\sigma: N \leftrightarrow N^{\prime}$ such that $P(i)=P^{\prime}(\sigma(i))$ for all $i \in N$, then we call $\bar{P}=\left(P, P^{\prime}\right)$ as a two-fold replica of $P$ and denote it by $2 P$. The definition naturally extends to all $c$-fold replicas $c P$ of $P$, for any $c \geq 2$ for $c \in \mathbb{N}$.

Given any finite $N \subsetneq \mathcal{N}$ and any $A \subsetneq \mathcal{A}$, a delegation rule $\varphi$ is a social welfare correspondence which assigns every preference profile $P \in \mathcal{L}(A)^{n}$ a nonempty subset of preferences $\varphi(P) \subseteq \mathcal{L}(A)$, interpreted as the set of delegates or the delegation for this society.

\subsection{Conditions}

Next, we introduce some conditions on how to choose a delegation. Unless otherwise mentioned, we assume the conditions to hold for all set of alternatives, $A \subsetneq \mathcal{A}$, and for all set of agents, $N \subsetneq \mathcal{N}$. The first condition requires that if everyone prefers an alternative over another, then no delegate should say otherwise.

Definition 1. Pareto optimality: A rule $\varphi$ is Pareto Optimal whenever for all $P \in \mathcal{L}(A)^{n}$ and for all $a, b \in A$, if for all $i \in N,(a, b) \in P(i)$, then for all $R \in \varphi(P),(a, b) \in R$.

The second condition we impose concerns merging of two societies each endowed with the same delegation. In such situations, the delegation assigned to the merged society should remain 
the same. This concept is well known in many contexts under varying names with slight changes, including reinforcement, homogeneity ${ }^{9}$, etc.

Definition 2. Consistency: A rule $\varphi$ is consistent whenever for any two disjoint finite sets $N, N^{\prime} \subsetneq \mathcal{N}$ (with cardinality $n$ and $n^{\prime}$ respectively) and for profiles, $P \in \mathcal{L}(A)^{n}$ and $P^{\prime} \in \mathcal{L}(A)^{n^{\prime}}$, if $\varphi(P)=\varphi\left(P^{\prime}\right)$ then $\varphi\left(\left(P, P^{\prime}\right)\right)=\varphi(P)=\varphi\left(P^{\prime}\right)$.

The third condition we impose concerns variable alternative scenarios, wherein the fixed set of individuals face more issues to report their preferences on. Consider, for instance, two sets of alternatives $A \subsetneq \bar{A}$ such that $|A|=3$ and $|\bar{A}|=4$. Consider two profiles on these sets with the following frequency supports:

$$
P \in \mathcal{L}(A)^{n} \text { with } p=(3,2,1,0,0,0) \text { and } \bar{P} \in \mathcal{L}(\bar{A})^{n} \text { with } \bar{p}=(0,1,2,3, \underbrace{0, \ldots, 0)}_{20 \text { entries }}
$$

Note that the nonzero entries in each vector are identical (except for the shuffling). Our condition requires that shuffling the support for preferences should shuffle the delegates in the exact same way. ${ }^{10}$ Formally, given $A \subseteq \bar{A} \subsetneq \mathcal{A}$, a profile $P \in \mathcal{L}(A)^{n}$ and an injection ${ }^{11} \pi$ : $\{1,2, \ldots,|A| !\} \rightarrow\{1,2, \ldots,|\bar{A}| !\}$, we say $\bar{P} \in \mathcal{L}(\bar{A})^{n}$ is an "expansion of $P$ by $\pi$ " if for all $i \in\{1,2, \ldots,|A| !\}$ we have $p_{i}=\bar{p}_{\pi(i)}$. We consider such profiles $P, \bar{P}$ to have equivalent ballots and call $\pi$ as a corresponding injection. ${ }^{12}$

Definition 3. Ballot neutrality: A rule is ballot neutral whenever for any $P \in \mathcal{L}(A)^{n}, \bar{P} \in$ $\mathcal{L}(\bar{A})^{n}$ with equivalent ballots, and for any corresponding injection $\pi$, we have:

$$
R_{i} \in \varphi(P) \text { if and only if } \bar{R}_{\pi(i)} \in \varphi(\bar{P}) \text {. }
$$

We provide an example in Appendix A.2 to demonstrate this condition. ${ }^{13}$ Next, we show that ballot neutrality, together with Pareto optimality, implies that the delegation can only be chosen from preferences that are reported. Thus we do not have to worry about finding a delegate whose role would be to represent some "compromised" preference. Let $R P(P)=\{R \in \mathcal{L}(A) \mid p(R)>0\}$, denote the set of reported preferences, preferences which are reported by at least one agent in profile $P$.

Proposition 2.1. If a rule $\varphi$ satisfies Pareto optimality and ballot neutrality, then for all $P \in$ $\mathcal{L}(A)^{n}, \varphi(P) \subseteq R P(P)$.

\footnotetext{
${ }^{9}$ Homogeneity is a milder version of this concept, which requires that result would be insensitive to replicating the population (Fishburn, 1977).

${ }^{10}$ This condition is, in fact, an amalgamation of two well-known axioms, neutrality and anonymity, and stronger than both.

${ }^{11}$ For $A=\bar{A}, \pi$ is a permutation.

${ }^{12}$ There may be more than one corresponding injection for two equivalent ballots.

${ }^{13}$ Note that the definition of ballot neutrality even extends to profiles on two disjoint sets of alternatives. For instance, let $A=\{x, y, z\}$ and $B=\{a, b, c\}$, and consider two profiles $P \in \mathcal{L}(A)^{n}$ and $\bar{P} \in \mathcal{L}(B)^{n}$ with identical ballots. Consider expansions of $P$ and $\bar{P}$, say $P^{\prime}$ and $\bar{P}^{\prime}$ respectively, to $A \cup B$ by some injection. Ballot neutrality applies between $P$ and $P^{\prime}$ (and between $\bar{P}$ and $\bar{P}^{\prime}$ ). By construction, $P^{\prime}$ and $\bar{P}^{\prime}$ have equivalent ballots. Therefore ballot neutrality applies between $P^{\prime}$ and $\bar{P}^{\prime}$. This, in turn, imposes ballot neutrality between $P$ and $\bar{P}$.
} 
Proof. The proof is in Appendix B.1.

The following remark says that we can always find an expansion for a profile in which delegates and non-delegates are clustered, that is, each agent whose preference is not in the delegation will prefer any non-delegate to any delegate. In other words, every agent who supports a preference which is not part of the delegation would like to enlarge the delegation set.

Let us extend our definition of injections to sets of preferences. Given $A \subseteq \bar{A} \subsetneq \mathcal{A}$ and any injection $\pi:\{1,2, \ldots|A| !\} \rightarrow\{1,2, \ldots|\bar{A}| !\}$, and any $X \subseteq \mathcal{L}(A)$,

$$
\pi(X)=\left\{\bar{R}_{\pi(i)} \in \mathcal{L}(\bar{A}) \mid R_{i} \in X\right\}
$$

Remark 2.1. Note that, since $\mathcal{A}$ is infinite, for any $A \subsetneq \mathcal{A}$, for any preference profile on $A$ and for any two disjoint sets $X, Y \subsetneq \mathcal{L}(A)$, we can always find an expansion $P$ by some $\pi$ of the initial preference profile such that the injections of the two sets $X$ and $Y$ (denoted respectively by $\pi(X)$ and $\pi(Y)$ ), form clusters that are "far away" from each other. Formally:

$$
\max _{R, R^{\prime} \in \pi(X)} \delta\left(R, R^{\prime}\right)<\min _{R \in \pi(X), R^{\prime} \in \pi(Y)} \delta\left(R, R^{\prime}\right)
$$

(The example in Appendix A.3 illustrates how this remark is implemented.)

Our third condition, strategy-proofness, implies that no agent should "benefit" from misreporting his preference, i.e., truth telling is a weakly dominant strategy. We say an agent $i$ weakly prefers a delegate $R_{1}$ to another delegate $R_{2}$, whenever $P(i)$ is weakly closer to $R_{1}$ than it is to $R_{2}$ in terms of the Kemeny distance, i.e., $\delta\left(P(i), R_{1}\right) \leq \delta\left(P(i), R_{2}\right)$. Similarly, we say an agent $i$ weakly prefers a delegation $D_{1}$ to another delegation $D_{2}$, whenever $P(i)$ is weakly closer to the most preferred delegate in $D_{1}$ than it is to the most preferred delegate in $D_{2}$, i.e., $\min \left\{\delta\left(P(i), R_{1}\right) \mid R_{1} \in D_{1}\right\} \leq \min \left\{\delta\left(P(i), R_{2}\right) \mid R_{2} \in D_{2}\right\}$. Strategy proofness means that every agent weakly prefers the delegation they get under true preferences to any delegation they achieve by misreporting. In other words, there is no possibility of misreporting and getting a new delegate in the delegation which is closer to the agent's preference. Here, we take the closest delegate as the only relevant one for the agents, meaning agents do not care about the distance to other delegates. ${ }^{14}$ We first discuss the usual individual strategy-proofness and afterward the coalitional version of it. In the sequel we shall only use the former, however, we show later in Proposition 2.2 that the latter is implied by the former under ballot neutrality.

Definition 4. Strategy-proofness: A rule $\varphi$ is strategy-proof whenever for all $i \in N$ and for all $P \in \mathcal{L}(A)^{n}$, there exists no $P^{\prime}=\left(P^{\prime}(i), P(N \backslash\{i\})\right) \in L(A)^{n}$ such that

$$
\min _{R \in \varphi(P)} \delta(P(i), R)>\min _{R \in \varphi\left(P^{\prime}\right)} \delta(P(i), R)
$$

\footnotetext{
${ }^{14}$ Here we do not assume any negative externality in representativeness, i.e., agents only care about the delegate(s) that are closest to them in terms of representation. Note, however, that other methods, e.g., averaging the distances to set $D$, or taking the median preference in $D$ would give perfectly valid but different scenarios of representation.
} 
Definition 5. Coalitional strategy-proofness: A rule is coalitional strategy-proof whenever for all coalitions $S \subseteq N$ and for all $P \in \mathcal{L}(A)^{n}$, there exists no $P^{\prime}=\left(P^{\prime}(S), P(N \backslash S)\right) \in \mathcal{L}(A)^{n}$ such that:

$$
\begin{gathered}
\min _{R \in \varphi(P)} \delta(P(i), R)>\min _{R \in \varphi\left(P^{\prime}\right)} \delta(P(i), R) \\
\text { for all } i \in S .
\end{gathered}
$$

Remark 2.2. Note that our individual strategy-proofness concept is essentially different than that of Bossert and Storcken (1992) and of Athanasoglou (2016). We allow multiple preferences in the outcome (in the case of single-valued delegation rules they are equivalent). A recent paper by Bossert and Sprumont (2014) differs from the former two interpretations since the manipulation is based on a concept known as betweenness (see also Grandmont (1978), Kemeny (1959), and Sato (2013)). In their interpretation, an agent can benefit only when the outcome is manipulated to somewhere between herself and the preference corresponding to truth telling. In our interpretation agents can benefit when the outcome is manipulated to anywhere, resulting a closer preference. This makes the strategy-proofness we propose, ceteris paribus, stronger and harder to satisfy. We provide an example in Appendix A.1 which is strategy-proof in the sense of Bossert and Sprumont (2014), but not in the way we interpret it.

Next, we show that under ballot neutrality strategy-proofness implies coalitional strategyproofness. We use this implication throughout the proofs.

Proposition 2.2. If a rule $\varphi$ is strategy-proof and ballot neutral, then it is also coalitional strategy-proof.

Proof. Let $\varphi$ be a strategy-proof and ballot neutral rule. For any $A \subsetneq \mathcal{A}$, any $N \subsetneq \mathcal{N}$ and any $P \in \mathcal{A}^{n}$, and for any $S \subseteq\{i \in N \mid P(i) \notin \varphi(P)\}$, let us denote any deviation from $P$ by agents in $S$ as $P^{\prime}=\left(P^{\prime}(S), P(N \backslash S)\right)$. Let $W=\varphi(P)$ and $O=\mathcal{L}(A) \backslash \varphi(P)$ denote a partition of $\mathcal{L}(A)$.

By Remark 2.1, there exists an expansion of $P$ by $\pi$, say $\bar{P}$, where $\bar{W}=\pi(W)$ and $\bar{O}=\pi(O)$ such that

$$
\max _{\bar{R}, R^{\prime} \in \bar{O}} \delta\left(\bar{R}, R^{\prime}\right)<\min _{\bar{R} \in \bar{O}, R^{\prime} \in \bar{W}} \delta\left(\bar{R}, R^{\prime}\right) .
$$

Consider any enumeration of $i \in S$, i.e. $S=\{1,2, \ldots, s\}$. Let us construct expanded profiles, $\bar{P}_{0}, \bar{P}_{1}, \ldots, \bar{P}_{s}$, with $\bar{P}_{0}=\bar{P}, \bar{P}_{s}=\bar{P}^{\prime}$ (the expansion of $P^{\prime}$ by $\pi$, i.e., $\bar{P}^{\prime}=\left(\bar{P}^{\prime}(S), \bar{P}(N \backslash S)\right.$ ), and for all $i \in\{1,2, \ldots, s\}, \bar{P}_{i}=\left(\bar{P}^{\prime}(\{1,2, \ldots, i\}), \bar{P}(N \backslash\{1,2, \ldots, i\})\right)$. This is a formalization of the idea that any deviation by a coalition can be constructed as a result of consecutive unilateral deviations by a sequence of agents.

By Proposition 2.1, $\varphi\left(\bar{P}_{i}\right) \subseteq R P\left(\bar{P}_{i}\right)$ for all $i \in\{1,2, \ldots, s\}$. Note that from $\bar{P}_{0}$ to $\bar{P}_{1}$, there cannot be a preference $\bar{R} \in \bar{O}$ that becomes a new delegate for $\bar{P}_{1}$. This is because by Inequality 2.1, we have that for all $R^{\prime} \in \bar{W}, \delta(\bar{R}, \bar{P}(1))<\delta\left(R^{\prime}, \bar{P}(1)\right)$ and this would contradict individual strategy proofness. A similar argument holds from $\bar{P}_{i}$ to $\bar{P}_{i+1}$ for any $i \in\{1,2, \ldots, s-$ $1\}$. As the choice of enumeration of agents in $S$ is arbitrary, eventually this implies that there 
exists no $\bar{R} \in \bar{O}$ such that $\bar{R} \in \varphi\left(\bar{P}_{s}\right)$. As $\bar{P}_{s}=\bar{P}^{\prime}$, and $\bar{P}^{\prime}$ is an expansion of $P^{\prime}$, then there exists no $R \in O$, such that $R \in \varphi\left(P^{\prime}\right)$, since there exists no $\bar{R} \in O$ with $\bar{R} \in \varphi\left(\bar{P}^{\prime}\right)$. Then $\varphi\left(P^{\prime}\right) \subseteq W=\varphi(P)$. As $\varphi\left(P^{\prime}\right)$ is a subset of $\varphi(P)$, this implies that no agent in $\mathrm{S}$ has become strictly better off, i.e.,

$$
\text { There exists no } i \in S \text { such that } \min _{R \in \varphi(P)} \delta(P(i), R)>\min _{R \in \varphi\left(P^{\prime}\right)} \delta(P(i), R) \text {. }
$$

Since all agents whose preferences are already included in the delegation, i.e, $P(i) \in W$ has distance of zero to the delegation, they will not have any incentive to deviate or to join a coalition. This means that there cannot be any coalition $S$ which can successfully manipulate.

\section{$3 \quad$ Using thresholds for delegation rules}

In this section, we introduce a large class of delegation rules which we call as threshold rules. Every threshold rule is associated with a particular threshold function which we introduce below. Thereafter we show that the rules are well-defined and provide some examples within this special class of delegation rules.

Definition 6. Threshold Function: A threshold function is a function denoted by $f: \mathbb{Z}^{+} \rightarrow$ $\left(\frac{1}{2}, 1\right] \cap \mathbb{Q}$ such that for all $t$ :

$$
f(t) \geq \frac{f(t-1)+1}{2} .
$$

These functions simply assign a threshold for each possible delegation of size $t$. Let us introduce some additional notation to define the threshold rules. Given any $P \in \mathcal{L}(A)^{n}$, consider an enumeration which orders preferences according to their support from the agents from the strongest to weakest, i.e., $p_{i} \geq p_{i+1}$. For example, $p=(0.5,0 . \overline{3}, 0.1 \overline{6}, 0,0, \ldots, 0)$ denotes the normalized support for $P$. Let us also denote the corresponding preferences as $R_{1}, R_{2}, \ldots, R_{|A|}$ i.e., $R_{1}$ is the preference with the strongest support and so forth. ${ }^{15}$ Then we can define the cumulative support $\rho$ as the cumulative vector of $p$, i.e., for all $i, \rho_{i}=p_{1}+\ldots+p_{i}$. For instance, the cumulative support for the aforementioned $P$ is: $\rho=(0.5,0.8 \overline{3}, 1,1, \ldots, 1)$.

We first introduce the threshold rules as an algorithm, then proceed with the formal definition.

Take any profile $P$ and the cumulative support for it as $\rho$. Consider any threshold function $f$. Let $R_{1}, R_{2}, \ldots R_{|A|}$ denote an ordering of preferences according to their support, with ties broken arbitrarily.

Step 1: Check whether $\rho_{1} \geq f(1)$. If yes, $\varphi^{f}(P)=\left\{R_{1}\right\}$ and the algorithm stops. Otherwise, go to the next step.

\footnotetext{
${ }^{15}$ Note that some preferences in profiles might have equal support with a tie. In that case, the enumeration of those preferences can be chosen arbitrarily.
} 
Step 2: Check whether $\rho_{2} \geq f(2)$. If yes, $\varphi^{f}(P)=\left\{R_{1}, R_{2}\right\}$ and the algorithm stops. Otherwise, go to the next step.

Step t: Check whether $\rho_{t} \geq f(t)$. If yes, $\varphi^{f}(P)=\left\{R_{1}, R_{2}, R_{3}, \ldots, R_{t}\right\}$ and the algorithm stops. Otherwise, go to the next step.

Note that the algorithm stops after finite steps since we are dealing with a finite subset $A$ of $\mathcal{A}$. Next, we propose the formal definition. Again, given any profile $P$, we use the enumeration $R_{1}, R_{2}, \ldots R_{|A|}$ which orders according to the size of the support.

Definition 7. Threshold Rule: Given a threshold function $f$ and $P \in \mathcal{L}(A)^{n}$, a threshold rule is $\varphi^{f}(P)=\left\{R_{1}, R_{2}, \ldots, R_{t^{*}}\right\}$ where $t^{*}=\underset{t}{\arg \min }\left\{t \in \mathbb{Z}^{+} \mid \rho_{t} \geq f(t)\right\}$.

Thus the threshold rule select the lowest number of delegates at which the relevant threshold for total support is reached. However, there are two immediate concerns about these delegations rules. The first is whether we can always find a delegation that exceeds the threshold. The second is what happens when the algorithm stops at $t^{*}$, where two preferences have equal support, i.e., $p_{t^{*}}=p_{t^{*}+1}$ and $R_{t^{*}} \in \varphi^{f}(P)$ but $R_{t^{*}+1} \notin \varphi^{f}(P)$. We address both concerns in Proposition 3.1 which shows that the rules are well-defined.

Proposition 3.1. For all $A \subsetneq \mathcal{A}, N \subsetneq \mathcal{N}, P \in \mathcal{L}(A)^{n}$, and all threshold functions $f$, the threshold rule $\varphi^{f}(P)$ is well-defined.

Proof. The proof is in Appendix 3.1.

Example 3.1. We will show four different delegation rules that are their threshold rules in the case of three alternatives. Let us consider the three different preference profiles, $P^{1}, P^{2}$, and $P^{3}$ denoted below by the normalized support (on the left side) and the cumulative support (on the right side) for preferences. Note that for the sake of simplicity we use profiles with the same enumeration wherein the support for $R_{i}$ is decreasing in $i$.

$$
\begin{array}{ll}
p^{1}=(0.31,0.29,0.29,0.11,0,0) & \rho^{1}=(0.31,0.6,0.89,1,1,1) \\
p^{2}=(0.78,0.12,0.1,0,0,0) & \rho^{2}=(0.78,0.9,1,1,1,1) \\
p^{3}=(0.55,0.12,0.11,0.11,0.11,0) & \rho^{3}=(0.55,0.67,0.78,0.89,1,1)
\end{array}
$$

The visualizations which is provided below capture the essence of threshold rules. Even though neither cumulative supports nor the relevant thresholds for each cardinality are actually continuous values, connecting discrete values via lines makes visualization easier. In the following graphs, the first number of delegates that a cumulative support is above the corresponding threshold indicates the number of delegates in the delegation. 
Throughout these examples, let us denote the threshold function as $f=(., \ldots, 1, \ldots)$, where the $i^{\text {th }}$ entry corresponds to $f(i)$. Since this function is increasing and has the bound of 1 , once the value of 1 is reached, all further values is equal to 1 .

- The first rule, $\varphi^{1}$ is defined by the threshold function $f^{1}=(0.51,0.76,0.89,0.95,1, \ldots)$. This rule checks whether the total support for some delegation reaches the relevant threshold for the size of the delegation, and if it does, picks that delegation with the smallest number of delegates.

- The second rule we deal with, $\varphi^{2}$ is characterized by the threshold vector, $f^{2}=(0.51,1, \ldots)$. This rule checks whether there exists any preference that is supported by more at least $51 \%$ of the agents and if it is the case makes it the singleton delegate. If it is not the case, the rule picks all reported preferences instead.

- The third rule we deal with, $\varphi^{3}$ is characterized by the threshold vector, $f^{3}=(0 . \overline{6}, 1, \ldots)$. This rule checks whether there exists any preference that is supported by more at least two-thirds of the agents (a.k.a. qualified majority), and if it is the case, then makes it the singleton delegate. If it is not the case, the rule picks all reported preferences instead.

- The last rule we deal with, $\varphi^{4}$ is the reported preference rule $R P(P)$, which chooses all preferences reported. The relevant threshold vector is $f^{4}=(1, \ldots)$, i.e. the total support for any delegation should be at least $100 \%$.

Visualizations of the rules and the delegations for each example profile is provided below. The bold numbers for $\rho_{i}$, indicates that $R_{i}$ is a chosen delegate for the profile $p$ under the rule $\varphi^{f}$.

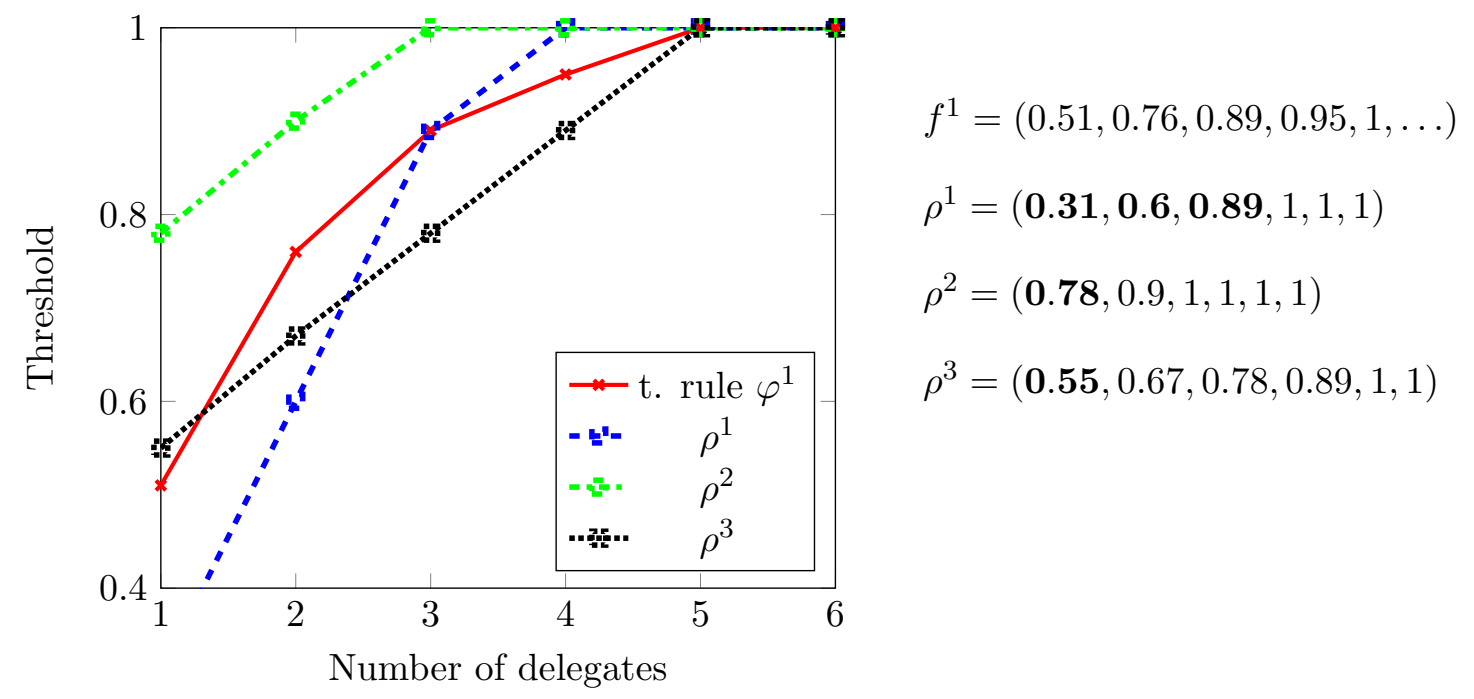

As it can be seen from the graph, $\varphi^{1}\left(P^{1}\right)=\left\{R_{1}, R_{2}, R_{3}\right\}$, while $\varphi^{1}\left(P^{2}\right)=\varphi^{1}\left(P^{3}\right)=\left\{R_{1}\right\}$. 


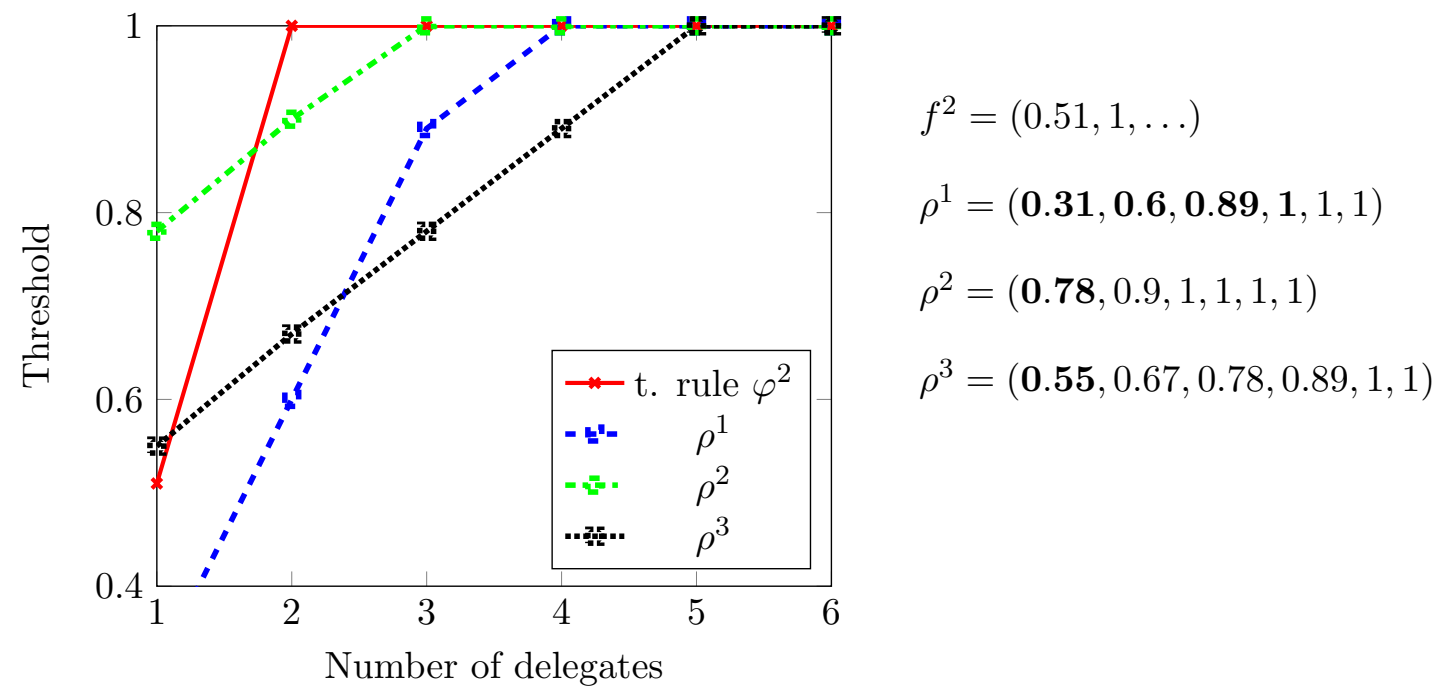

As it can be seen from the graph, $\varphi^{2}\left(P^{1}\right)=\left\{R_{1}, R_{2}, R_{3}, R_{4}\right\}$, while $\varphi^{2}\left(P^{2}\right)=\varphi^{2}\left(P^{3}\right)=\left\{R_{1}\right\}$.

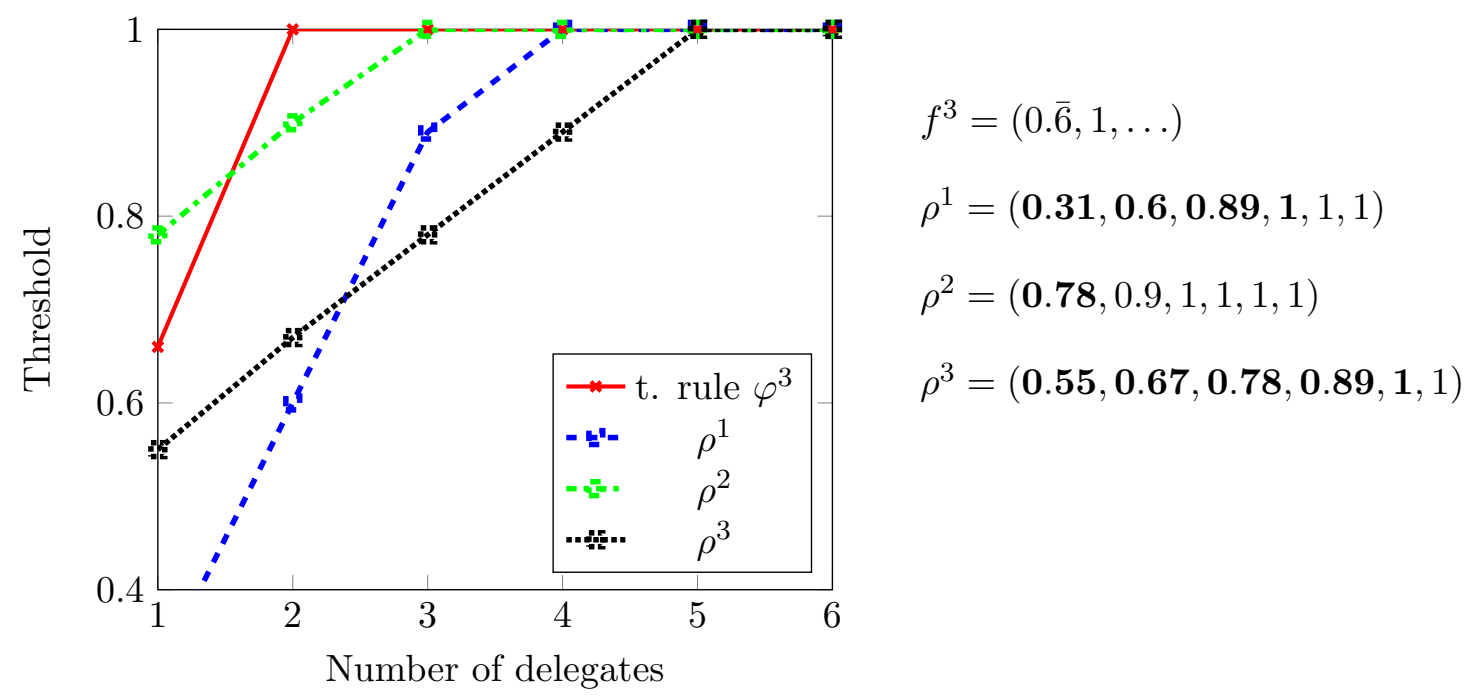

As it can be seen from the graph, $\varphi^{3}\left(P^{1}\right)=\left\{R_{1}, R_{2}, R_{3}, R_{4}\right\}, \varphi^{3}\left(P^{2}\right)=\left\{R_{1}\right\}$, and $\varphi^{3}\left(P^{3}\right)=$ $\left\{R_{1}, R_{2}, R_{3}, R_{4}, R_{5}\right\}$. 


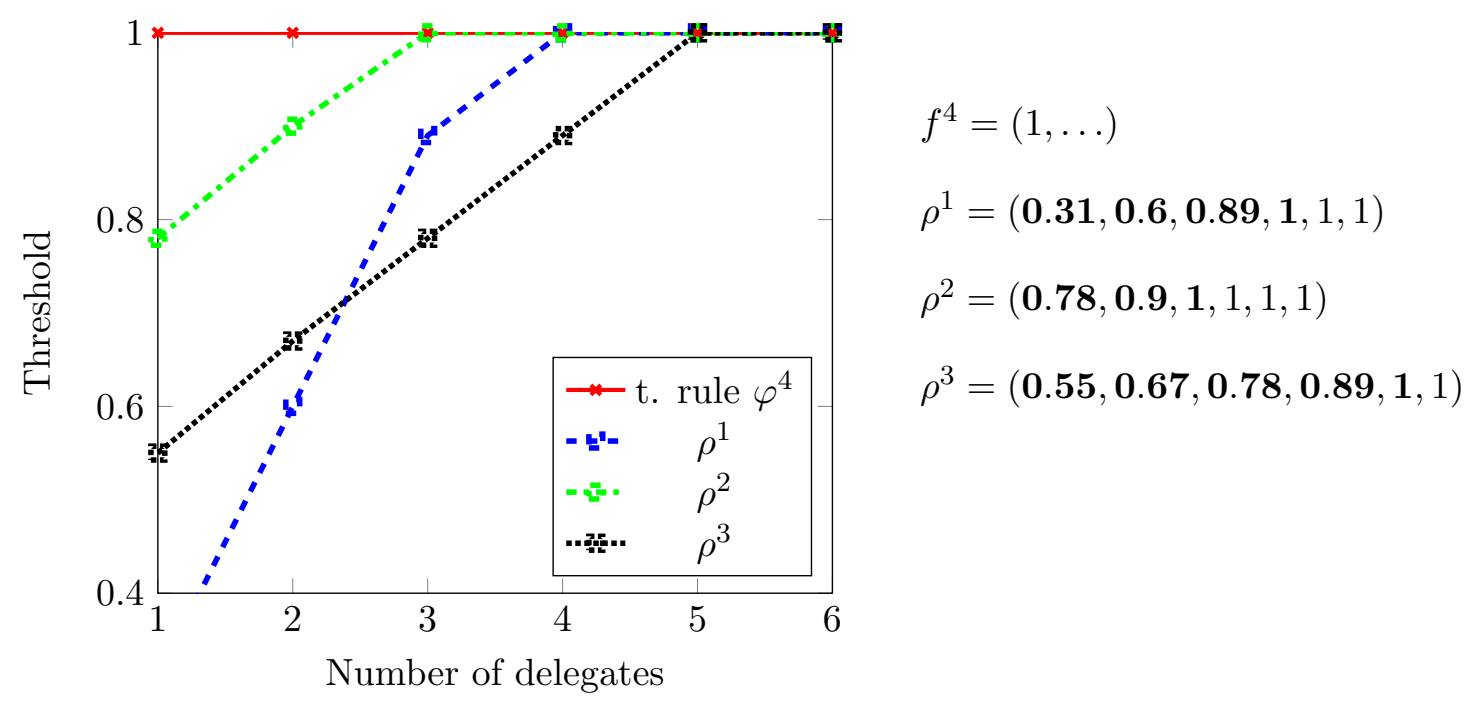

As it can be seen from the graph, $\varphi^{4}\left(P^{1}\right)=\left\{R_{1}, R_{2}, R_{3}, R_{4}\right\}, \varphi^{4}\left(P^{2}\right)=\left\{R_{1}, R_{2}, R_{3}\right\}$, and $\varphi^{4}\left(P^{3}\right)=\left\{R_{1}, R_{2}, R_{3}, R_{4}, R_{5}\right\}$.

\section{Characterization of the threshold delegation rules}

In this section, we show that the conditions of Pareto optimality, consistency, ballot neutrality, and strategy-proofness characterize the class of delegation rules which we explained in the previous section. We show that these conditions lead to some implications concerning the behaviour of the delegation rules. The first four lemmas shape the structure of the rules concerning the support of delegates. Another four lemmas prove the existence of a series of critical thresholds for choosing delegates and set forth the structure of these thresholds. We conclude the section with our main theorem which states that the only rules satisfying the conditions we demand are the threshold delegation rules.

\subsection{Delegates and their support in the society}

In what follows, Lemma 4.1 shows that if a preference is chosen as a delegate, then any other preference with stronger support in the society should also be chosen. Lemma 4.2 argues that rules should only care about the percentage of the support, i.e., only the normalized support of preference profiles matter. Lemma 4.3 proves that i) equal redistribution of the total support for the delegates among themselves does not change the delegation, and ii) equal redistribution of the total support for the rest among themselves also does not modify the delegation.

Lemma 4.1. If a rule $\varphi$ satisfies consistency, ballot neutrality, and strategy-proofness, then for all $A \subsetneq \mathcal{A}, N \subsetneq \mathcal{N}$ and $P \in \mathcal{L}(A)^{n}$ if $R \in \varphi(P)$ and $p\left(R^{\prime}\right) \geq p(R)$, we have $R^{\prime} \in \varphi(P)$.

Proof. The proof is in Appendix B.3. 
This lemma and Proposition 2.1 implies that any rule satisfying these conditions will have a delegation composed of preferences with relatively higher support compared to preferences that are not in the delegation. The following lemma proves the delegations to be the same for two different societies with identical normalized supports. Let $p / n=\left(\frac{p_{1}}{n}, \frac{p_{2}}{n}, \ldots, \frac{p_{|A| !}}{n}\right)$ denote the normalized support.

Lemma 4.2. If a rule $\varphi$ satisfies consistency and ballot neutrality, then for all $A \subsetneq \mathcal{A}, N, N^{\prime} \subsetneq \mathcal{N}$ and for all $P \in \mathcal{L}(A)^{n}, P^{\prime} \in \mathcal{L}(A)^{n^{\prime}}$ such that $p / n=p^{\prime} / n^{\prime}$, we have $\varphi(P)=\varphi\left(P^{\prime}\right)$.

Proof. The proof is in Appendix B.4.

The following lemma proves that neither averaging between supports of chosen delegates nor averaging between supports of non-delegates will change the delegation.

Lemma 4.3. If a rule $\varphi$ satisfies consistency and ballot neutrality, then for all $A \subsetneq \mathcal{A}, N \subsetneq \mathcal{N}$ and $P \in \mathcal{L}(A)^{n}$, denoting $|\varphi(P)|=t$, and picking an enumeration on $\mathcal{L}(A)$ such that $p_{i} \geq p_{j}$ for all $i<j$, the following holds:

i) For any $N^{\prime} \subsetneq \mathcal{N}$ for any $P^{\prime} \in \mathcal{L}(A)^{n^{\prime}}$ such that $\frac{p_{j}^{\prime}}{n^{\prime}}=\sum_{i=1}^{t} \frac{p_{i}}{n t}$ for all $j \in\{1,2, \ldots, t\}$ and $\frac{p_{j}^{\prime}}{n^{\prime}}=\frac{p_{j}}{n}$ for all $j \in\{t+1, t+2, \ldots,|A| !\}$ we have $\varphi(P)=\varphi\left(P^{\prime}\right)$.

ii) For any $N^{\prime \prime} \subsetneq \mathcal{N}$ and for any $P^{\prime \prime} \in \mathcal{L}(A)^{n^{\prime \prime}}$ such that $\frac{p_{j}^{\prime \prime}}{n^{\prime \prime}}=\frac{p_{j}}{n}$ for all $j \in\{1,2, \ldots, t\}$ and $\frac{p_{j}^{\prime \prime}}{n^{\prime \prime}}=\sum_{i=t+1}^{|A| !} \frac{p_{j}}{n(|A| !-t)}$ for all $j \in\{t+1, t+2, \ldots,|A| !\}$ we have $\varphi(P)=\varphi\left(P^{\prime \prime}\right)$.

Proof. The proof is in Appendix B.5.

Example 4.1. As an example for those two cases, let us take some $A$ with $|A|=3$. Let us take $P \in \mathcal{L}(A)^{n}$ with support $p=(\mathbf{8}, \mathbf{7}, \mathbf{6}, 3,0,0)$ where bold numbers indicate the support for the chosen delegates. As an example for two subcases of the Lemma 4.3, let us take $P^{\prime}, P^{\prime \prime} \in \mathcal{L}(A)^{n}$ with supports $p^{\prime}=(7,7,7,3,0,0), p^{\prime \prime}=(8,7,6,1,1,1)$ respectively. Then, Lemma 4.3 implies $\varphi(P)=\varphi\left(P^{\prime}\right)=\varphi\left(P^{\prime \prime}\right)=\left\{R_{1}, R_{2}, R_{3}\right\}$.

Remark 4.1. Using permutations and merging as in the proof of Lemma 4.3, it is straightforward to see that the lemma applies also to any subset of delegates or non-delegates. That is, averaging between supports of some subset of chosen delegates or some subset of non-delegates will not change the delegation.

In the next lemma, we show that if a preference is chosen as a delegate, it must have more support than the total support for all the preferences which are not in the delegation. This is mainly due to the strategy-proofness condition.

Lemma 4.4. If a rule $\varphi$ satisfies ballot neutrality and strategy-proofness, then for all $A \subsetneq \mathcal{A}$, $N \subsetneq \mathcal{N}$, and $P \in \mathcal{L}(A)^{n}$ if $R \in \varphi(P)$, then we have

$$
p(R)>\sum_{R^{\prime} \notin \varphi(P)} p\left(R^{\prime}\right) .
$$

Proof. The proof is in Appendix B.6. 


\subsection{When to choose a delegate, and when not to?}

As seen in the previous four lemmas, any well-defined delegation rule satisfying conditions of Pareto optimality, consistency, ballot neutrality, and strategy-proofness takes the most supported preference in the delegation. However, for this preference to be the only delegate, it has to be really powerful, i.e., powerful enough to eliminate all the other opinions. We need a new tool to capture this. Take any $\varphi$ which satisfies all the conditions. Categorize all $P \in \mathcal{L}(A)^{n}$ for any $N \subsetneq \mathcal{N}$ and $A \subsetneq \mathcal{A}$ according to the size of the delegations as follows: $\mathcal{P}_{t}=\left\{P \in \mathcal{L}(A)^{n}|| \varphi(P) \mid=t\right\}$. Lemma 4.1 and 4.2 imply that we only have to focus on the normalized support of the profiles from stronger to the weaker. Therefore, we can define a vector for this $\varphi$ for any $A \subsetneq \mathcal{A}$ as

$$
k^{\varphi}(A)=\left[k_{1}, k_{2}, \ldots, k_{|A| !}\right], \text { where each } k_{t}=\min _{P \in \mathcal{P}_{t}}\left(\sum_{i=1}^{t} p_{i}\right) / n .
$$

To ease the notation, we will omit $\varphi$ from $k^{\varphi}$ whenever it is clear. Furthermore, by ballot neutrality, we know that for these rules the vector $k$ is the same for every $A$ with equal cardinality. To understand these vectors, consider all profiles which end up with a single delegate under $\varphi$. Then $k_{1}$ gives us the relative support of the delegate with minimal value, among all the profiles with a single delegation. Similarly $k_{i}$ gives the total relative support of the delegation with the minimal value, among all the profiles with a delegation of size $i$. In what follows, we discuss some features of these vectors.

Lemma 4.5 shows how $k_{i}$ values relate to one another. Lemma 4.6 shows if a preference has more relative support than $k_{1}$ it has to be chosen uniquely. Lemma 4.7 shows how the choice of delegates depends on $k$ in general. Finally, Lemma 4.8 shows how the vectors for sets of alternatives of different sizes relate to each other.

Lemma 4.5. If a rule $\varphi$ satisfies Pareto optimality, consistency, ballot neutrality, and strategyproofness, then for all $A \subsetneq \mathcal{A}$, and $N \subsetneq \mathcal{N}$ the corresponding vector satisfies that $k_{t}^{\varphi}(A) \geq$ $\frac{k_{t-1}^{\varphi}(A)+1}{2}$ for all $t \in\{2,3, \ldots,|A| !\}$.

Proof. The proof is in Appendix B.7.

In the following two lemmas, Lemma 4.6 and 4.7, we assume an enumeration which orders preferences according to their support from the agents from the strongest to weakest, i.e., $p_{i} \geq p_{j}$ for all $i<j$. Lemma 4.6 shows that for profiles in which a strongest single preference has a relative support $p_{1} / n$ more than $k_{1}$, the delegation should only consist of this preference, $R_{1}$. Lemma 4.7 extends this to larger delegation sizes, i.e., the delegation should comprise of the first $t$ strongest preferences whose relative total support surpasses their corresponding threshold $k_{t}$ while no smaller subdelegation satisfies this.

Lemma 4.6. If a rule $\varphi$ satisfies Pareto optimality, consistency, ballot neutrality, and strategyproofness, then for all $A \subsetneq \mathcal{A}, N \subsetneq \mathcal{N}$ and for any $P \in \mathcal{L}(A)^{n}$ such that $p_{1} \geq n k_{1}^{\varphi}(A)$, we have that $\varphi(P)=\left\{R_{1}\right\}$. 
Proof. The proof is in Appendix B.8.

Lemma 4.7. If a rule $\varphi$ satisfies Pareto optimality, consistency, ballot neutrality, and strategyproofness, then for all $A \subsetneq \mathcal{A}, N \subsetneq \mathcal{N}$ and for any $P \in \mathcal{L}(A)^{n}$ such that

i) for some $t>1, \sum_{i=1}^{t} p_{i} \geq n k_{t}^{\varphi}(A)$ and,

ii) for all $l<t, \sum_{i=1}^{l} p_{i}<n k_{l}^{\varphi}(A)$

we have: $\varphi(P)=\left\{R_{1}, R_{2}, \ldots, R_{t}\right\}$.

Proof. The proof is in Appendix B.9.

The next lemma shows that the corresponding vectors of the rules are independent of the number of alternatives.

Lemma 4.8. If a rule $\varphi$ satisfies ballot neutrality, then for any $A \subsetneq \bar{A} \subsetneq \mathcal{A}$, the corresponding vector satisfies that $k^{\varphi}(A)_{t}=k^{\varphi}(\bar{A})_{t}$ for all $t \in\{1,2, \ldots,|A| !\}$.

Proof. The proof is in Appendix B.10.

Note that Lemma 4.8 has further implications. In fact, for any two sets of alternatives, $A, B$ the $k^{\varphi}(A)_{t}$ and $k^{\varphi}(B)_{t}$ values will always correspond to each other. That can be achieved by extending each of the sets to $A \cup B$ by separately by implementing the lemma above.

Next, our main theorem finalizes the result by showing there is only one class of delegation rules, i.e., the threshold rules, associated with a threshold function that satisfies all the conditions we have imposed.

Theorem 4.1. A rule $\varphi$ satisfies Pareto optimality, consistency, ballot neutrality, and strategyproofness if and only if for all $A \subsetneq \mathcal{A}, N \subsetneq \mathcal{N}$ and $P \in \mathcal{L}(A)^{n}$ we have that $\varphi(P)=\varphi^{f}(P)$ for some threshold function $f$.

Proof. We leave the if part to the reader and prove the only if part. Proposition 2.1 and Lemma 4.1 together imply that only preferences with higher support will be assigned as delegates as opposed to those with lower support. Lemma 4.2 implies that the degree of this relative power will be independent of the number of agents. Lemma 4.3 states that averaging of supports for delegates will not change the delegation. This implies that only the normalized supports for the delegations matter.

Given any rule $\varphi$ satisfying the conditions, we can then construct a corresponding vector $k(A)$ where the $i^{\text {th }}$ entry is the minimum support needed for a delegation of size $i$ across all preference profiles on $A$. By consistency, $k$ is constant across all possible subsets of agents $N \subsetneq \mathcal{N}$, and by Lemma $4.8, k(A)_{i}=k(B)_{i}$, i.e., $k$ is also constant across all possible sets of alternatives $A \subsetneq \mathcal{A}$. Hence, regardless of the domain of the delegation rule, one can construct a function $f$ on positive 
integers such that $f(i)=k_{i}$ for all possible delegations of size $i$. By Lemma 4.4, we have that $f(1)>1 / 2$. By Lemma 4.5 , for all $t>1$ we have that

$$
f(t) \geq \frac{f(t-1)+1}{2} .
$$

Thus, by construction, $f: \mathbb{Z}^{+} \rightarrow\left(\frac{1}{2}, 1\right] \cap \mathbb{Q}$ is the unique threshold function induced by $\varphi$ as in Definition 6, and hence $\varphi=\varphi^{f}$ is a threshold delegation rule.

\section{Conclusion}

This paper brings about a novel class of rules for choosing a delegation, characterized by intuitive fairness, efficiency, and non-manipulability properties. The nature of these threshold delegation rules is such that they provide a good compromise in at least three aspects, inclusivity, minimalism, and non-manipulability. Inclusivity is often deemed as crucial since it results in the legitimacy of the political settlement (Dudouet and Lundström (2016)). Minimalism, in the sense that not everyone can be invited to the table, is also an important parameter of simplicity of design in conflict resolution. Finally, non-manipulability of a delegation rule is essential so that people's true opinions are always reflected in the conflict resolution, preventing further re-escalation of post-truce conflicts.

The rules we characterize share one important feature in common. If a single delegate is sent by the rule to the table, then that delegate, and the opinion she represents, must have strictly more than $50 \%$ support in the society. Let us consider a more demanding threshold rule which requires a $60 \%$ for a single delegate representation. In that case, the minimal required support for a delegation of size 2 becomes at least $80 \%$ (averaging $60 \%$ and 100\%). The thresholds for larger delegations quickly increase by averaging each threshold with $100 \%$ to find the next threshold, making it harder for small delegations to reach. Therefore, these rules are fairly inclusive in most of the cases. In addition to that, the more fragmented the society gets, the more inclusive the rules become.

As hinted in the introduction, the lower bound of all the threshold rules follow a well-known elementary geometric series related to Zeno's Dichotomy (somehow also related to Aristotle's story on Achilles and the Tortoise). Let $t$ denote the size of the delegation, and $f$ denote a threshold function. Then all the threshold functions obey the following lower bounds:

$$
f(t)>\frac{1}{2}+\left(\frac{1}{2}\right)^{2}+\ldots+\left(\frac{1}{2}\right)^{t}=\sum_{i=1}^{t}\left(\frac{1}{2}\right)^{i} .
$$

There are directions that we foresee for future research concerning the delegation choice. For instance, our selection of minimal Kemeny distance as a measure of well-being of agents leads to a particular definition of strategy-proofness, which implies non-externality in representation. That is, we define a manipulation to be beneficial for an individual when the individual can successfully alter the "minimal distance" to the delegation. Of course, one might define manipulation as 
successfully altering the "average distance" to the delegation instead, which includes externality in representation, i.e., introducing additional delegates might reduce the well-being of agents.

Another direction is the choice of metric used to define strategy-proofness. Although Kemeny distance is quite standard in the literature, one might employ other metrics to see the robustness of these findings. We would like to note, however, that none of our proofs uses the properties of Kemeny distance specifically. As long as the clustering result in Remark 2.1 can be reached, we expect that our results can be generalized. Perhaps a minimal set of common features/axioms satisfied by any metric will lead to similar results. We suspect one of this particular axioms to be the betweenness axiom introduced in Kemeny $(1959)^{16}$.

Another direction for future research is about our selection of minimal Kemeny distance as a measure of well-being of agents. If the assumption of nonexternality in representation is modified, and also reversed to see implications of different assumptions with the setup. One obvious way would be to assume that agents do try to minimize the distance to the farthest delegate, or to the mean of the delegation. In the most extreme generalization, one may also assume that each agent has different ways of deciding between different delegations.

We conclude our paper by suggesting the policy makers that next time a peace delegation of size $t$ is summoned from different opinions, then the ratio of uninvited opinions should not account more than $(0.5)^{t}$ of the population, e.g., if only 3 delegates are invited, then uninvited groups should account for less than $12.5 \%$ of the population. This gives us a minimal bound for inclusivity, furthermore can help us to quantify "negligible minorities".

\footnotetext{
${ }^{16}$ Betweenness requires that if three rankings are on a line, i.e., the middle one also agrees on all pairwise issues that has been agreed by the other two, then the distance should be additive.
} 


\section{References}

Athanasoglou, S. (2016). Strategyproof and efficient preference aggregation with kemeny-based criteria. Games and Economic Behavior 95, 156 - 167.

Barberà, S. (2011). Strategyproof social choice, Chapter 25, pp. 731-831. Elsevier.

Barberà, S., B. Dutta, and A. Sen (2001). Strategy-proof social choice correspondences. Journal of Economic Theory 101(2), 374-394.

Bossert, W. and Y. Sprumont (2014). Strategy-proof preference aggregation: Possibilities and characterizations. Games and Economic Behavior 85(1), 109-126.

Bossert, W. and T. Storcken (1992). Strategy-proofness of social welfare functions: The use of the kemeny distance between preference orderings. Social Choice and Welfare, 345-360.

Can, B. and A. J. A. Storcken (2013). A re-characterization of the kemeny distance. GSBE Research Memorandum 9.

Dudouet, V. and S. Lundström (2016). Post-war political settlements: From participatory transition processes to inclusive state-building and governance. Research report, Berghof Foundation, Berlin.

Fishburn, P. C. (1977). Condorcet social choice functions. SIAM Journal on Applied Mathematics 33(3), 469-489.

Gibbard, A. (1973). Manipulation of voting schemes: a general result. Econometrica: journal of the Econometric Society, 587-601.

Grandmont, J.-M. (1978). Intermediate preferences and the majority rule. Econometrica $46(2)$, $317-330$.

Kemeny, J. G. (1959). Mathematics without numbers. Daedalus 88(4), 577-591.

Lanz, D. (2011). Who gets a seat at the table? a framework for understanding the dynamics of inclusion and exclusion in peace negotiations. International Negotiation 16(2), 275-295.

Papagianni, K. (2014). National dialogue processes in political transitions. Discussion paper no. 3, Civil Society Dialogue Network.

Planta, K., V. Prinz, and L. Vimalarajah (2015). Inclusivity in national dialogues - guaranteeing social integration or preserving old power hierarchies? inclusive political settlements. Research report, Berghof Foundation, Berlin.

Sato, S. (2013). A sufficient condition for the equivalence of strategy-proofness and nonmanipulability by preferences adjacent to the sincere one. Journal of Economic Theory 148(1), 259-278. 
Satterthwaite, M. A. (1975). Strategy-proofness and arrow's conditions: Existence and correspondence theorems for voting procedures and social welfare functions. Journal of economic theory $10(2), 187-217$.

Smith, J. H. (1973). Aggregation of preferences with variable electorate. Econometrica: Journal of the Econometric Society, 1027-1041.

Young, H. P. (1974). An axiomatization of borda's rule. Journal of economic theory 9(1), 43-52.

Young, H. P. (1975). Social choice scoring functions. SIAM Journal on Applied Mathematics $28(4), 824-838$.

\section{A Examples}

\section{A.1 Example for showing the Kemeny rule is not strategy-proof}

Example A.1. Given a profile $P \in \mathcal{L}(A)^{n}$, a preference $R$ is a Kemeny ranking for $\mathrm{P}$, if for all $R^{\prime} \in \mathcal{L}(A)$, we have that $\sum_{i \in N} \delta(R, P(i)) \leq \sum_{i \in N} \delta\left(R^{\prime}, P(i)\right)$. A rule which assigns all Kemeny rankings to each profile is called the Kemeny rule. More formally, the Kemeny rule, denoted by $\varphi_{K}$, assigns to a profile $P \in \mathcal{L}(A)^{n}: \varphi_{K}(P)=\{R \in L(A) \mid \mathrm{R}$ is a Kemeny ranking for $\mathrm{P}\}$.

Our counterexample is just with 4 alternatives, and 11 agents. $P$ is as follows:

$\begin{array}{lllllllllll}d & d & d & a & c & c & a & b & b & b & b \\ a & a & a & d & a & a & b & c & c & c & c \\ b & b & c & c & b & b & d & d & d & d & a \\ c & c & b & b & d & d & c & a & a & a & d\end{array}$

It can be seen that $\varphi(P)=\{a b c d\} . \quad \delta(a b c d, b c a d)=2$. The last agent can manipulate to reach the following $P^{\prime}$ :

$\begin{array}{lllllllllll}d & d & d & a & c & c & a & b & b & b & b \\ a & a & a & d & a & a & b & c & c & c & c \\ b & b & c & c & b & b & d & d & d & d & d \\ c & c & b & b & d & d & c & a & a & a & a\end{array}$

It can be seen that $\varphi\left(P^{\prime}\right)=\{b c d a\} . \delta(b c d a, b c a d)=1$. So, the last agent is in a better position with reporting a false profile. So, even though both the Kemeny rule and our strategyproofness condition is defined upon minimal Kemeny distance, we show that the Kemeny rule is not strategy-proof.

\section{A.2 Example for a rule which is Ballot Neutral}

Example A.2. Let us take two sets, $A$ and $B$ with $A \subsetneq \bar{A},|A|=3$ and $|\bar{A}|=4$. Let us have 7 agents. For this example, we use the lexicographic enumeration where alternatives are ordered 
with their place in the alphabet, so $R_{1}=a b c, R_{2}=a c b$ and so on, and $\bar{R}_{1}=a b c d, \bar{R}_{2}=a b d c$ and so on.

Let us define $P \in \mathcal{L}(A)^{7}$ as

$\begin{array}{lllllll}a & a & a & a & a & b & b \\ b & b & b & c & c & a & c \\ c & c & c & b & b & c & a\end{array} \quad$ Here, $p=(3,2,1,1,0,0)$.

Take some $\varphi$ where $\varphi(P)=\{a b c\}=\left\{R_{1}\right\}$. Next, we define $\bar{P} \in \mathcal{L}(\bar{A})^{7}$ as

$\begin{array}{lllllll}a & a & a & a & a & a & a \\ b & b & b & b & b & c & c \\ c & c & c & d & d & b & d \\ d & d & d & c & c & d & b\end{array} \quad$ Here, $\bar{p}=(3,2,1,1, \underbrace{0 \ldots 0}_{20 \text { zeros. }})$.

There is an injective function from $A$ to $\bar{A}$ that satisfies our condition. For $\pi(t)=t$, we have $p_{t}=\bar{p}_{\pi(t)}=\bar{p}_{t}$ for all $t \in\{1,2, \ldots, 6\}$. So, for $\varphi$ to satisfy ballot neutrality, we must have $\varphi(\bar{P})=\left\{\bar{R}_{\pi(1)}\right\}=\left\{\bar{R}_{1}\right\}=\{a b c d\}$.

Or, since ballot neutrality is binding two ways, we can claim that if $\varphi(\bar{P})=\{a b c d\}=$ $\left\{\bar{R}_{\pi(1)}\right\}=\left\{\bar{R}_{1}\right\}$, then we must have that $\varphi(P)=\{a b c\}=\left\{R_{1}\right\}$.

\section{A.3 Example for a profile in which ballot neutrality is used to increase coali- tional options.}

Example A.3. Consider $A=\{a, b, c\}, N=16$, and a profile $P$ whose support is $p=(5,4,3,2,1,1)$. If $\varphi(P)=\left\{R_{1}, R_{2}, R_{3}, R_{4}\right\}$, then agents whose preferences are not in the delegation have distance of 1 to the nearest delegate in all possible permutations. So, there is not a possible clustering, in which every agent strictly prefers some other non-delegate to the closest delegate for this particular alternative set. However, when we move to $\bar{A}=\{a, b, c, d\}$, it is easy to see that there is such a cluster with correct injection.

\section{B Proofs}

\section{B.1 Proof of Proposition 2.1}

Proposition 2.1. If a rule $\varphi$ satisfies Pareto optimality and ballot neutrality, then for all $P \in$ $\mathcal{L}(A)^{n}, \varphi(P) \subseteq R P(P)$.

Proof: Take some finite $A \subsetneq \mathcal{A}$ and $N \subsetneq \mathcal{N}$. Take some preference profile $P \in \mathcal{L}(A)^{n}$ with $R P(P) \subseteq \mathcal{L}(A)$. First, note that with equality, we are done. Suppose, for a contradiction, that there exists a preference $R \in \varphi(P)$ with $p(R)=0$ that is, a preference with zero support. Consider any $x \in \mathcal{A} \backslash A$ and let us construct the expansion of $P$ to $\bar{A}=A \cup x$ by $\bar{P} \in \mathcal{L}(\bar{A})^{n}$ as follows: for all $i \in N, \bar{P}(i)=P(i) \| x$ where $P(i) \| x=P(i) \cup(x, x) \cup\{(a, x) \mid a \in A\}$, i.e., concatenation of $x$ 
with $P(i)$. Clearly, for any $a \in A$ and $i \in N,(a, x) \in \bar{P}(i)$. Take any $a \in A$, and $R^{*} \in \mathcal{L}(\bar{A})$ such that $(x, a) \in R^{*}$. By Pareto optimality, $R^{*} \notin \varphi(\bar{P})$. Note that $\bar{p}\left(R^{*}\right)=0=\bar{p}(R \| x)$. Then by ballot neutrality $R \| x \notin \varphi(\bar{P})$. As $\bar{P}$ is an expansion of $P$, again by ballot neutrality, we conclude that $R \notin \varphi(P)$.

\section{B.2 Proof of Proposition 3.1}

Proposition 3.1. For all $A \subsetneq \mathcal{A}, N \subsetneq \mathcal{N}, P \in \mathcal{L}(A)^{n}$, and all threshold functions $f$, the threshold rule $\varphi^{f}(P)$ is well-defined.

Proof: Let us pick some threshold function $f$, and consider the corresponding threshold rule $\varphi^{f}(P)$. To show that the rule is well-defined, we need to show the following.

\section{There is always a $t$ that satisfies $\rho_{t} \geq f(t)$.}

For any $p \in \mathcal{L}(A)^{n}, t=|A|$ !, satisfies this. Since any reported preference should be within $\mathcal{L}(A)$, including all preferences in the delegation ensures to have cumulative support of 1 . Since by definition any $f(t) \leq 1$, we have: $\rho_{|A| !} \geq f(|A| !)$. This shows that the universal set is always guaranteed to pass the relevant threshold.

\section{There is always a unique way to pick the first $t$ preferences.}

Case 1: For all distinct $i, j$, we have $p_{i} \neq p_{j}$.

If this is the case, powers of the preferences are well ordered, there is a unique ordering enumeration, so there is always a unique way to pick the first $t$ preferences.

Case 2: For some distinct $i, j$, we have $p_{i}=p_{j}$.

We will show that for any $p_{i}=p_{j}$ it is either $R_{i}, R_{j} \in \varphi^{f}(P)$ or $R_{i}, R_{j} \notin \varphi^{f}(P)$.

First, consider the case where the number of delegates is one. In that case, we must have $\rho_{1}=\frac{p_{1}}{n} \geq f(1)>\frac{1}{2}$. This directly shows that any enumeration has the same preference as its first, whenever the first preference is passing the relevant threshold.

Second, consider the case when $t^{*}>1$. By definition, for all $t>1$ we have that

$$
\begin{array}{rr}
f(t) \geq \frac{1+f(t-1)}{2} . & \text { Multiply both sides by } 2 \text { to get } \\
2 f(t) \geq 1+f(t-1) . & \text { Subtract } f(t)+f(t-1) \text { from both sides to get } \\
& f(t)-f(t-1) \geq 1-f(t) .
\end{array}
$$

From definition of the rule, we know the following is true for some $t^{*}$ :

$$
\begin{aligned}
\rho_{t^{*}-1} & <f\left(t^{*}-1\right), \\
\rho_{t^{*}} & \geq f\left(t^{*}\right) .
\end{aligned}
$$

Multiplying both sides of B.2b by -1 and adding 1 to both sides we get

$$
1-\rho_{t^{*}} \leq 1-f\left(t^{*}\right)
$$

Subtracting B.2a from B.2b leads to 


$$
\frac{p\left(t^{*}\right)}{n}>f\left(t^{*}\right)-f\left(t^{*}-1\right)
$$

If we combine B.3, B.4, and B.1 we get

$$
\frac{p\left(t^{*}\right)}{n}>f\left(t^{*}\right)-f\left(t^{*}-1\right) \geq 1-f\left(t^{*}\right) \geq 1-\rho_{t^{*}}=\sum_{i=t^{*}+1}^{|A| !} \frac{p_{i}}{n} .
$$

The rightmost term is the total support for the preferences which are not part of the delegation, where the leftmost term is the support for the weakest delegate. This implies that not only the weakest delegate has strictly more support than the next preference, but he also has strictly more support than the total support for non-delegates. This says that there is again a unique way to select the topmost $t$ preferences as delegates, even though the relevant enumeration is not unique this time. In other words, when there is any tie between support for some preferences, by design all of those preferences belong to the delegation or none of them.

\section{B.3 Proof of Lemma 4.1}

Lemma 4.1. If a rule $\varphi$ satisfies consistency, ballot neutrality, and strategy-proofness, then for all $A \subsetneq \mathcal{A}, N \subsetneq \mathcal{N}$ and $P \in \mathcal{L}(A)^{n}$ if $R \in \varphi(P)$ and $p\left(R^{\prime}\right) \geq p(R)$, we have $R^{\prime} \in \varphi(P)$.

Proof: Suppose, for a contradiction, that there exists two preferences $R^{\mathrm{h}}$ (preference with (h)igher support), and $R^{\mathrm{l}}$ (preference with (l)ower support) with $R^{\mathrm{l}} \in \varphi(P)$ and $R^{\mathrm{h}} \notin \varphi(P)$. Let $p\left(R^{\mathrm{h}}\right)=h$ and $p\left(R^{\mathrm{l}}\right)=l$. Without loss of generality, we can assume that $h+l$ is even since by consistency we can replicate the profile once by using the two-fold replica with no changes in the delegation. This will ensure that $h-l$ can also be assumed to be even.

First, assume that $h-l=0$. This contradicts ballot neutrality since $h=l$. Next, assume that $h-l=2$. In that case, an agent whose original preference is $R^{\mathrm{h}}$ may misreport $R^{\mathrm{l}}$. Denoting the modified profile by $P^{\prime}$, that will cause $p^{\prime}\left(R^{\mathrm{h}}\right)=p^{\prime}\left(R^{\mathrm{l}}\right)$. From ballot neutrality, either both of

$R^{\mathrm{h}}$ and $R^{\mathrm{l}}$ will be in the delegation, or none will be included. If both are included, this means that the agent deviated to his benefit, contradicting strategy-proofness. If none is included, some agent with $R^{\mathrm{l}}$ as his original preference may report $R^{\mathrm{h}}$ to get back to the original preference profile, resulting in $R^{\mathrm{l}} \in \varphi(P)$ again, this also contradicts strategy-proofness. So for $h-l=2$, we showed that with the original preference profile, if $R^{\mathrm{l}}$ is included in the delegation, so must $R^{\mathrm{h}}$ be.

Assume that our hypothesis holds for $h-l=k$ for some even $k$, that is, if $R^{l} \in \varphi(P)$, then $R^{\mathrm{h}} \in \varphi(P)$. Now, let $h-l=k+2$. Then an agent whose original preference is $R^{\mathrm{h}}$ can report $R^{\mathrm{l}}$ to trigger the situation with $h-l=k$. Since this violates strategy-proofness, we must have $R^{\mathrm{h}} \in \varphi(P)$ even when $h-l=k+2$. By induction, this completes the proof.

\section{B.4 Proof of Lemma 4.2}

Lemma 4.2. If a rule $\varphi$ satisfies consistency and ballot neutrality, then for all $A \subsetneq \mathcal{A}, N, N^{\prime} \subsetneq \mathcal{N}$ and for all $P \in \mathcal{L}(A)^{n}, P^{\prime} \in \mathcal{L}(A)^{n^{\prime}}$ such that $p / n=p^{\prime} / n^{\prime}$, we have $\varphi(P)=\varphi\left(P^{\prime}\right)$. 
Proof: From consistency, we know that $\varphi(P)=\varphi(2 P)=\varphi(3 P)=\ldots=\varphi(n P)$. So, $\varphi(P)=$ $\varphi\left(n^{\prime} P\right)$ and $\varphi\left(P^{\prime}\right)=\varphi\left(n P^{\prime}\right)$. Since $n^{\prime} p=n p^{\prime}$, ballot neutrality implies that $\varphi\left(n^{\prime} P\right)=\varphi\left(n P^{\prime}\right)$, completing the proof.

\section{B.5 Proof of Lemma 4.3}

Lemma 4.3. If a rule $\varphi$ satisfies consistency and ballot neutrality, then for all $A \subsetneq \mathcal{A}, N \subsetneq \mathcal{N}$ and $P \in \mathcal{L}(A)^{n}$, denoting $|\varphi(P)|=t$, and picking an enumeration on $\mathcal{L}(A)$ such that $p_{i} \geq p_{j}$ for all $i<j$, the following holds:

i) For any $N^{\prime} \subsetneq \mathcal{N}$ for any $P^{\prime} \in \mathcal{L}(A)^{n^{\prime}}$ such that $\frac{p_{j}^{\prime}}{n^{\prime}}=\sum_{i=1}^{t} \frac{p_{i}}{n t}$ for all $j \in\{1,2, \ldots, t\}$ and $\frac{p_{j}^{\prime}}{n^{\prime}}=\frac{p_{j}}{n}$ for all $j \in\{t+1, t+2, \ldots,|A| !\}$ we have $\varphi(P)=\varphi\left(P^{\prime}\right)$.

ii) For any $N^{\prime \prime} \subsetneq \mathcal{N}$ and for any $P^{\prime \prime} \in \mathcal{L}(A)^{n^{\prime \prime}}$ such that $\frac{p_{j}^{\prime \prime}}{n^{\prime \prime}}=\frac{p_{j}}{n}$ for all $j \in\{1,2, \ldots, t\}$ and $\frac{p_{j}^{\prime \prime}}{n^{\prime \prime}}=\sum_{i=t+1}^{|A| !} \frac{p_{j}}{n(|A| !-t)}$ for all $j \in\{t+1, t+2, \ldots,|A| !\}$ we have $\varphi(P)=\varphi\left(P^{\prime \prime}\right)$.

Proof: 1. Let $P$ and $p=\left(p_{1}, p_{2}, \ldots, p_{|A| !}\right)$ be as in the Lemma with $|\varphi(P)|=t$ and $P^{\prime}$ as defined in the Lemma. Consider the following profiles with the same enumeration on $\mathcal{L}(A)$ where $p_{1}, p_{2}, \ldots, p_{t}$ rotates and bold numbers indicate the support for the chosen delegates:

$$
\begin{gathered}
P^{1} \in \mathcal{L}(A)^{n} \text { such that } p^{1}=\left(\mathbf{p}_{\mathbf{t}}, \mathbf{p}_{\mathbf{1}}, \mathbf{p}_{\mathbf{2}}, \ldots, \mathbf{p}_{\mathbf{t}-\mathbf{1}}, p_{t+1}, p_{t+2}, \ldots, p_{|A| !}\right) \\
P^{2} \in \mathcal{L}(A)^{n} \text { such that } p^{2}=\left(\mathbf{p}_{\mathbf{t}-\mathbf{1}}, \mathbf{p}_{\mathbf{t}}, \mathbf{p}_{\mathbf{1}}, \ldots, \mathbf{p}_{\mathbf{t}-\mathbf{2}}, p_{t+1}, p_{t+2}, \ldots, p_{|A| !}\right) \\
\vdots \\
P^{t-1} \in \mathcal{L}(A)^{n} \text { such that } p^{t-1}=\left(\mathbf{p}_{\mathbf{2}}, \mathbf{p}_{\mathbf{3}}, \mathbf{p}_{\mathbf{4}}, \ldots, \mathbf{p}_{\mathbf{1}}, p_{t+1}, p_{t+2}, \ldots, p_{|A| !}\right)
\end{gathered}
$$

From ballot neutrality, we know that $\varphi(P)=\varphi\left(P^{i}\right)$ for any $i \in\{1, \ldots, t-1\}$. By design, merging all these profiles $\left(P, P^{1}, P^{2}, \ldots, P^{t-1}\right)$ gives us $t P^{\prime}$, and from consistency, we get that $\varphi\left(t P^{\prime}\right)=\varphi(P)$. From Lemma $4.2 \varphi(P)=\varphi\left(t P^{\prime}\right)=\varphi\left(P^{\prime}\right)$ is guaranteed.

2. Let $P$ and $p=\left(p_{1}, p_{2}, \ldots, p_{|A| !}\right)$ be as in the Lemma with $|\varphi(P)|=t$ and $P^{\prime \prime}$ as defined in the Lemma. Consider the following profiles with the same enumeration on $\mathcal{L}(A)$ where $p_{t+1}, p_{t+2}, \ldots, p_{|A|}$ ! rotates and bold numbers indicate the support for the chosen delegates:

$$
\begin{gathered}
P^{1} \in \mathcal{L}(A)^{n} \text { such that } p^{1}=\left(\mathbf{p}_{\mathbf{1}}, \mathbf{p}_{\mathbf{2}}, \ldots, \mathbf{p}_{\mathbf{t}}, p_{|A| !}, p_{t+1}, p_{t+2}, \ldots, p_{|A| !-1}\right) \\
P^{2} \in \mathcal{L}(A)^{n} \text { such that } p^{2}=\left(\mathbf{p}_{\mathbf{1}}, \mathbf{p}_{\mathbf{2}}, \ldots, \mathbf{p}_{\mathbf{t}}, p_{|A| !-2}, p_{|A| !}, p_{t+1}, \ldots, p_{|A| !-2}\right) \\
\vdots \\
P^{t-1} \in \mathcal{L}(A)^{n} \text { such that } p^{t-1}=\left(\mathbf{p}_{\mathbf{1}}, \mathbf{p}_{\mathbf{2}}, \ldots, \mathbf{p}_{\mathbf{t}}, p_{t+2}, p_{t+3}, p_{t+4}, \ldots, p_{t+1}\right)
\end{gathered}
$$


From ballot neutrality, we know that $\varphi(P)=\varphi\left(P^{i}\right)$ for any $i \in\{1, \ldots, t-1\}$. By design, merging all these profiles $\left(P, P^{1}, P^{2}, \ldots, P^{t-1}\right)$ gives us $t P^{\prime \prime}$, and from consistency we get that $\varphi\left(t P^{\prime \prime}\right)=\varphi(P)$. From Lemma $4.2 \varphi(P)=\varphi\left(t P^{\prime \prime}\right)=\varphi\left(P^{\prime \prime}\right)$ is guaranteed.

\section{B.6 Proof of Lemma 4.4}

Lemma 4.4. If a rule $\varphi$ satisfies ballot neutrality and strategy-proofness, then for all $A \subsetneq \mathcal{A}$, $N \subsetneq \mathcal{N}$, and $P \in \mathcal{L}(A)^{n}$ if $R \in \varphi(P)$, then we have

$$
p(R)>\sum_{R^{\prime} \notin \varphi(P)} p\left(R^{\prime}\right) .
$$

Proof: Let us denote by $W=\varphi(P)$ the preferences of "winning" agents, and by $L=R P(P) \backslash$ $\varphi(P)$ the preferences of "losing" agents, where $R P(P)=\{R \in \mathcal{L}(A) \mid p(R)>0\}$ is again the set of reported preferences. Suppose, for a contradiction, that there exists a profile $P \in \mathcal{L}(A)^{n}$ and a preference $R_{t} \in \varphi(P)$ such that:

$$
p\left(R_{t}\right) \leq \sum_{\bar{R} \in L} p(\bar{R}) .
$$

That is, a preference $R_{t}$ in the delegation has weakly less support than the total support for all preferences of losing agents combined. By Remark 2.1, there exists an expansion of $P$, in which the corresponding injections of $W$ and $L$ are clustered far away from each other. By the same logic, one can find an expansion by $\pi$, say $\bar{P}$, if needed to an even larger alternative set, in which in addition to having $\pi(W)$ and $\pi(L)$ as far away clusters, $R_{\pi(t)}$ is relatively closer to $\pi(L)$ than to the other preferences in $\pi(W)$. Formally:

$$
\max _{R, R^{\prime} \in \pi(L)} \delta\left(R, R^{\prime}\right)<\min _{R \in \pi(L), R^{\prime} \in \pi(W)} \delta\left(R, R^{\prime}\right)
$$

and for any $R, R^{\prime} \in \pi(L), \tilde{R} \in \pi(W)$,

$$
\delta\left(R, R^{\prime}\right)<\delta\left(R, R_{\pi(t)}\right)<\delta(R, \tilde{R})
$$

Now consider a transformation of this expansion, denoted by $\bar{P}^{\prime}$, where all losing agents concentrate on a preference of a fellow losing agent, say $R^{s}$. By construction:

$$
\sum_{R \in L} p(R)=\bar{p}^{\prime}\left(R^{s}\right)
$$

By supposition, $\bar{p}\left(R_{\pi(t)}\right) \leq \bar{p}\left(R^{s}\right)$. Note that by coalitional strategy proofness, we have $R^{s} \notin \varphi\left(\bar{P}^{\prime}\right)$. Then we have two cases:

Case 1: If $R_{\pi(t)} \in \varphi\left(\bar{P}^{\prime}\right)$, then by Lemma $4.1, R^{s}$ should also be in the delegation $\varphi\left(\bar{P}^{\prime}\right)$, which is a contradiction. 
Case 2: If $R_{\pi(t)} \notin \varphi\left(\bar{P}^{\prime}\right)$, furthermore by Inequality B.7, $R_{\pi(t)}$ is a favorable preference for all agents with $\bar{P}(i) \in \pi(L)$. Then the agents in $L$ can misreport (and disperse back to their preferences in $\bar{P})$. As $R_{\pi(t)} \in \varphi(\bar{P})$, this contradicts strategy proofness.

\section{B.7 Proof of Lemma 4.5}

Lemma 4.5. If a rule $\varphi$ satisfies Pareto optimality, consistency, ballot neutrality, and strategyproofness, then for all $A \subsetneq \mathcal{A}$, and $N \subsetneq \mathcal{N}$ the corresponding vector satisfies that $k_{t}^{\varphi}(A) \geq$ $\frac{k_{t-1}^{\varphi}(A)+1}{2}$ for all $t \in\{2,3, \ldots,|A| !\}$.

Proof: Let us start with some $A \subsetneq \mathcal{A}$ and some $t<|A|$ ! -1 . Using consistency, we can pick some $N \subsetneq \mathcal{N}$ with $|N|=n$ divisible by all numbers up to $t+1$ without loss of generality. Let us take a specific $P \in \mathcal{L}(A)^{n}$, which is defined as $p_{i}=a$ for all $i \in\{1,2, \ldots, t+1\}$ and $p_{i}=0$ for $i \in\{t+2, t+3, \ldots,|A|$ ! $\}$. From Proposition 2.1 and ballot neutrality, we know that the delegation includes only the first $t+1$ preferences. Let us denote this profile as (with bold numbers indicating the support for the chosen delegates)

$$
p=(\underbrace{\mathbf{a}, \mathbf{a}, \ldots, \mathbf{a}}_{t+1 \text { times }}, \underbrace{0,0, \ldots, 0}_{|A| !-t-1 \text { times }}) .
$$

Now, let us deal with a modification $P^{\prime} \in \mathcal{L}(A)^{n}$ for this profile which is defined as $p_{i}^{\prime}=a^{\prime}=$ $\frac{n k_{t}}{t}$ for all $i \in\{1,2, \ldots, t\}, p_{t+1}^{\prime}=b=n\left(1-k_{t}\right)$ and $p_{i}^{\prime}=0$ for $i \in\{t+2, t+3, \ldots,|A| !\}$. Since the first $t$ preferences have $k_{t}$ support in total, by definition of $k_{t}$ and by Lemma 4.3 , we know that the delegation includes only the first $t$ preferences. Let us denote this profile as

$$
p^{\prime}=(\underbrace{\mathbf{a}^{\prime}, \mathbf{a}^{\prime}, \ldots, \mathbf{a}^{\prime}}_{t \text { times }}, b, \underbrace{0,0, \ldots, 0}_{|A| !-t-1 \text { times }}) .
$$

Another relevant modification of this profile, $P^{\prime \prime} \in \mathcal{L}(A)^{n}$ will be defined as $p_{i}^{\prime \prime}=a^{\prime \prime}=\frac{n-2 b}{t-1}$ for all $i \in\{1,2, \ldots, t\}, p_{i}^{\prime \prime}=b=n\left(1-k_{t}\right)$ for $i \in\{t, t+1\}$ and $p_{i}^{\prime \prime}=0$ for $i \in\{t+2, t+3, \ldots,|A| !\}$, e.g. $p^{\prime \prime}=\left(a^{\prime \prime}, a^{\prime \prime}, \ldots, a^{\prime \prime}, b, b, 0,0, \ldots, 0\right)$. By Proposition 2.1 we have that $\varphi\left(P^{\prime \prime}\right) \subseteq R P(P)$, and by ballot neutrality either i) $\varphi\left(P^{\prime \prime}\right)=\left\{R_{1}, R_{2}, \ldots, R_{t+1}\right\}$ or ii) $\varphi\left(P^{\prime \prime}\right)=\left\{R_{1}, R_{2}, \ldots, R_{t-1}\right\}$. Suppose, for a contradiction, that the former is the case. Let us take the average of support for the first $t$ preferences to get $P^{\prime}$. By Remark 4.1, this should not change the delegation. However, $\varphi\left(P^{\prime}\right)=\left\{R_{1}, R_{2}, \ldots, R_{t}\right\} \neq\left\{R_{1}, R_{2}, \ldots, R_{t+1}\right\}=\varphi\left(P^{\prime \prime}\right)$, which is a contradiction. So, it must be that ii) is the case, $\varphi\left(P^{\prime \prime}\right)=\left\{R_{1}, R_{2}, \ldots, R_{t-1}\right\}$. Let us denote this profile as

$$
p^{\prime \prime}=(\underbrace{\mathbf{a}^{\prime \prime}, \mathbf{a}^{\prime \prime}, \ldots, \mathbf{a}^{\prime \prime}}_{t-1 \text { times }}, b, b, \underbrace{0,0, \ldots, 0}_{|A| !-t-1 \text { times }}) .
$$

By definition, $k_{t-1}$ is the minimal support for all delegations with size $t-1$. Since only the first $t-1$ preferences are in the delegation, total support for the first $t-1$ preferences could be at least $k_{t-1}$. Then, $(t-1) a^{\prime \prime}=(t-1) \frac{n-2 b}{t-1}=n\left(1-2\left(1-k_{t}\right)\right) \geq n k_{t-1}$. After rearranging we get that 


$$
k_{t} \geq \frac{k_{t-1}+1}{2}
$$

\section{B.8 Proof of Lemma 4.6}

Lemma 4.6. If a rule $\varphi$ satisfies Pareto optimality, consistency, ballot neutrality, and strategyproofness, then for all $A \subsetneq \mathcal{A}, N \subsetneq \mathcal{N}$ and for any $P \in \mathcal{L}(A)^{n}$ such that $p_{1} \geq n k_{1}^{\varphi}(A)$, we have that $\varphi(P)=\left\{R_{1}\right\}$.

Proof: Let $P^{*} \in \mathcal{L}(A)^{n^{*}}$ be one of the profiles where $\left|\varphi\left(P^{*}\right)\right|=1$ and $p_{1}^{*}=n^{*} k_{1}^{\varphi}(A)$, i.e., one of the profiles wherein only a single delegate is assigned whose relative support defines $k_{1}$ in the corresponding vector. Consider now any $P \in \mathcal{L}(A)^{n}$ with $p_{1} \geq n k_{1}^{\varphi}(A)$. By Lemma 4.1, $R_{1} \in \varphi(P)$ and by ballot neutrality we can assume that $\varphi\left(P^{*}\right)=\left\{R_{1}\right\}$, i.e., the strongest ranking is the same both in $P$ and $P^{*}$. Next, we show that $R_{1}$ is the only delegate assigned to $P$, i.e., $\left\{R_{1}\right\}=\varphi(P)$.

By consistency, we can replicate profiles $P$ and $P^{*}$ ( $n^{*}$ and $n$ times respectively) with no changes in the delegation. With abuse of notation, let us denote these replicated profiles by $P, P^{*} \in \mathcal{L}(A)^{n \times n^{*}}$. So, we have $p_{1}^{*}=n n^{*} k_{1}^{\varphi}(A)$ and $p_{1} \geq n n^{*} k_{1}^{\varphi}(A)$.

Suppose, for a contradiction, that $\varphi(P) \supsetneq\left\{R_{1}\right\}$, so there is another delegate, say $R_{k}$ in the delegation. Let us partition $\mathcal{L}(A)$ into two sets, $X=\mathcal{L}(A) \backslash\left\{R_{1}\right\}$ and $Y=\left\{R_{1}\right\}$. By Remark 2.1, there exists an expansion of $P$ by $\pi$, say $\bar{P}$, in which the injection of $X$, i.e., $\pi(X)$ is clustered far away from the injection of $R_{1}$, i.e., $R_{\pi(1)}$. Formally:

$$
\max _{R, R^{\prime} \in \pi(X)} \delta\left(R, R^{\prime}\right)<\min _{R \in \pi(X)} \delta\left(R, R_{\pi(1)}\right)
$$

Note that $R_{\pi(1)}=\pi(Y)$ and $R_{\pi(k)} \in \pi(X)$. Let $\bar{P}^{*}$ denote the expansion of $P^{*}$ by the same injection, $\pi$. By ballot neutrality, i) $R_{\pi(k)} \notin \varphi\left(\bar{P}^{*}\right)$, implying $k^{\text {th }}$ strongest preference of $\bar{P}^{*}$ is not in the delegation of $\bar{P}^{*}$, and ii) $R_{\pi(k)} \in \varphi(\bar{P})$, implying that the $k^{\text {th }}$ strongest preference of $\bar{P}$ is in the delegation of $\bar{P}$. Note that as $\bar{p}_{1}^{*} \leq \bar{p}_{1}$, from $\bar{P}^{*}$ to $\bar{P}$ this means that there is a coalition of agents moving from $\pi(X)$ to $R_{\pi(1)}$, resulting in $R_{\pi}(k) \in \varphi(\bar{P})$. As $R_{\pi(k)} \in \pi(X)$, by Inequality B.8, this contradicts coalitional strategy proofness. Hence $R_{\pi(k)} \notin \varphi(\bar{P})$. Ballot neutrality then implies that $R_{k} \notin \varphi(P)$.

\section{B.9 Proof of Lemma 4.7}

Lemma 4.7. If a rule $\varphi$ satisfies Pareto optimality, consistency, ballot neutrality, and strategyproofness, then for all $A \subsetneq \mathcal{A}, N \subsetneq \mathcal{N}$ and for any $P \in \mathcal{L}(A)^{n}$ such that

i) for some $t>1, \sum_{i=1}^{t} p_{i} \geq n k_{t}^{\varphi}(A)$ and,

ii) for all $l<t, \sum_{i=1}^{l} p_{i}<n k_{l}^{\varphi}(A)$ 
we have: $\varphi(P)=\left\{R_{1}, R_{2}, \ldots, R_{t}\right\}$.

Proof: Take any $P \in \mathcal{L}(A)^{n}$ as defined in the lemma. As $\sum_{i=1}^{l} p_{i}<n k_{l}$ for all $l<t$, by definiton of the corresponding vector, we have $|\varphi(P)| \neq l$. This means that $|\varphi(P)| \geq t$. By Lemma 4.1 we get that $\varphi(P) \supseteq\left\{R_{1}, R_{2}, \ldots, R_{t}\right\}$. Next, we show that $\varphi(P)=\left\{R_{1}, R_{2}, \ldots, R_{t}\right\}$.

By consistency, we can assume that $\sum_{i=1}^{t} p_{i}$ is divisible by $t$ without loss of generality. By Remark 4.1, we can take the average support for the first $t$ preferences without changing the delegation. Let us denote this modified profile by $P^{\prime}$, which is defined as $p_{i}^{\prime}=a^{\prime}=\sum_{l=1}^{t} \frac{p_{l}}{t}$ if $i \leq t$ and $p_{i}^{\prime}=p_{i}$ if $i>t$.

Let $P^{*} \in \mathcal{L}(A)^{n^{*}}$ be one of the profiles where $\left|\varphi\left(P^{*}\right)\right|=t, \sum_{i=1}^{t} p_{i}^{*}=n^{*} k_{t}$, i.e., one of the profiles wherein only the strongest $t$ delegates are assigned whose relative total support defines $k_{t}$ in the corresponding vector.

By consistency, we can assume that $\sum_{i=1}^{t} p_{i}^{*}$ is divisible by $t$ without loss of generality. By Remark 4.1, we can take the average support for the first $t$ preferences without changing the delegation. Let us denote this modified profile also by $P^{*}$, where $p_{i}^{*}=a=\frac{n^{*} k_{t}}{t}$ for all $i \leq t$.

Using consistency, we can replicate profiles $P^{\prime}$ and $P^{*}\left(n^{*}\right.$ and $n$ times respectively) with no changes in the delegation. With abuse of notation, let us denote these replicated profiles by $P^{\prime}, P^{*} \in \mathcal{L}(A)^{n \times n^{*}}$. By construction, the total support for the strongest $t$ preferences in $P^{\prime}$ is larger than those in $P^{*}$, i.e., $n^{*} a^{\prime} t \geq$ nat.

Suppose, for a contradiction, that $\varphi\left(P^{\prime}\right) \supsetneq\left\{R_{1}, R_{2}, \ldots, R_{t}\right\}$ so there is another delegate, say $R_{k}$ with $k>t$ in the delegation. Let us partition $\mathcal{L}(A)$ into two sets, $X=\mathcal{L}(A) \backslash\left\{R_{1}, R_{2}, \ldots, R_{t}\right\}$ and $Y=\left\{R_{1}, R_{2}, \ldots, R_{t}\right\}$. By Remark 2.1, there exists an expansion of $P^{\prime}$ by $\pi$, say $\bar{P}^{\prime}$, in which the injection of $Y$ is clustered far away from the injection of $X$. Formally:

$$
\max _{R, R^{\prime} \in \pi(X)} \delta\left(R, R^{\prime}\right)<\min _{R \in \pi(X), R^{\prime} \in \pi(Y)} \delta\left(R, R^{\prime}\right)
$$

Note that $\left\{R_{\pi(1)}, R_{\pi(2)}, \ldots, R_{\pi(t)}\right\}=\pi(Y)$ and $R_{\pi(k)} \in \pi(X)$. Let $\bar{P}^{*}$ denote the expansion of $P^{*}$ by the same injection, $\pi$. By ballot neutrality, $R_{\pi(k)} \notin \varphi\left(\bar{P}^{*}\right)$ while $R_{\pi(k)} \in \varphi\left(\bar{P}^{\prime}\right)$. Note that from $\bar{P}^{*}$ to $\bar{P}^{\prime}$ there is a coalition of agents moving from $\pi(X)$ to $\pi(Y)$, resulting in $R_{\pi}(k) \in \varphi\left(\bar{P}^{\prime}\right)$. As $R_{\pi(k)} \in \pi(X)$, by Inequality B.9, this contradicts coalitional strategy proofness.

\section{B.10 Proof of Lemma 4.8}

Lemma 4.8. If a rule $\varphi$ satisfies ballot neutrality, then for any $A \subsetneq \bar{A} \subsetneq \mathcal{A}$, the corresponding vector satisfies that $k^{\varphi}(A)_{t}=k^{\varphi}(\bar{A})_{t}$ for all $t \in\{1,2, \ldots,|A| !\}$.

Proof: We denote corresponding threshold vectors as

$$
k^{\varphi}(A)=\left[k_{1}, k_{2}, \ldots, k_{|A| !}\right]
$$




$$
k^{\varphi}(\bar{A})=\left[k_{1}^{\prime}, k_{2}^{\prime}, \ldots, k_{|\bar{A}| !}^{\prime}\right]
$$

Suppose, for a contradiction, that there exist $A, \bar{A} \subsetneq \mathcal{A}$ such that $A \subsetneq \bar{A}$ with for some $t \in\{1,2, \ldots,|A| !\}, k_{t} \neq k_{t}^{\prime}$ while for all $i \in\{1,2, \ldots, t-1\}, k_{i}=k_{i}^{\prime}$. Without loss of generality, assume that $k_{t}<k_{t}^{\prime}$.

Then we construct a profile $P \in \mathcal{L}(A)^{n}$ and an expansion of $P$, by some $\pi$, denoted by $\bar{P}$ such that $\rho_{t}>k_{t}, \rho_{t}<k_{t}^{\prime}$ and $\rho_{i}<k_{i}$ for all $i \in\{1,2, \ldots, t-1\}$.

By Lemma 4.6 and Lemma 4.7 we have that $\varphi(P)=\left\{R_{1}, R_{2}, \ldots, R_{t}\right\}$, while $\varphi\left(P^{\prime}\right) \supsetneq$ $\left\{R_{\pi(1)}, R_{\pi(2), \ldots, R_{\pi(t)}}\right\}$. Since $\varphi\left(P^{\prime}\right)$ includes other elements than the image of $\varphi(P)$ under $\pi$, this contradicts ballot neutrality.

\section{Independence of Axioms}

The conditions used in the characterization were: Pareto optimality, consistency, strategy-proofness, ballot neutrality. Below, to put forward the logical independence of those, let us take a look at the following four social welfare correspondences.

- All but Pareto optimality: $\varphi(P)=\mathcal{L}(A)$ for any $P \in \mathcal{L}(A)^{n}$.

- All but consistency:

$$
\varphi(P)= \begin{cases}R, & \text { if } n \text { is odd and } \exists R \text { with } p(R)>\frac{N}{2} \\ R, & \text { if } n \text { is even and } \exists R \text { with } p(R)>\frac{2 N}{3} \\ R P(P), & \text { otherwise. }\end{cases}
$$

- All but strategy-proofness: $\varphi(P)=\left\{R \mid p(R) \geq p\left(R^{\prime}\right)\right.$ for all $\left.R^{\prime} \in \mathcal{L}(A)\right\}$.

- All but ballot neutrality:

$$
\varphi(P)= \begin{cases}\mathcal{L}(A), & \text { if }|\mathcal{L}(A) \backslash R P(P)|=1 \\ R P(P), & \text { otherwise. }\end{cases}
$$

